\title{
Diffusion-jump model for the combined Brownian and Neel relaxation dynamics of ferrofluids in the presence of external fields and flow
}

Article

Accepted Version

Ilg, P. (2019) Diffusion-jump model for the combined Brownian and Neel relaxation dynamics of ferrofluids in the presence of external fields and flow. Physical Review E, 100 (2). 022608. ISSN 1539-3755 doi:

https://doi.org/10.1103/PhysRevE.100.022608 Available at https://centaur.reading.ac.uk/85701/

It is advisable to refer to the publisher's version if you intend to cite from the work. See Guidance on citing.

To link to this article DOI: http://dx.doi.org/10.1103/PhysRevE.100.022608

Publisher: American Physical Society

All outputs in CentAUR are protected by Intellectual Property Rights law, including copyright law. Copyright and IPR is retained by the creators or other copyright holders. Terms and conditions for use of this material are defined in the End User Agreement. 


\section{CentAUR}

Central Archive at the University of Reading

Reading's research outputs online 


\title{
Diffusion-jump model for the combined Brownian and Néel relaxation dynamics of ferrofluids in the presence of external fields and flow
}

\author{
Patrick Ilg \\ School of Mathematical, Physical, and Computational Sciences, \\ University of Reading, Reading, RG6 6AX, United Kingdom
}

(Dated: August 20, 2019)

\begin{abstract}
Relaxation of suspended magnetic nanoparticles occurs via Brownian rotational diffusion of the particle as well as internal magnetization dynamics. The latter is often modeled by the stochastic Landau-Lifshitz equation, but its numerical treatment becomes prohibitively expensive in many practical applications due to a time-scale separation between fast, Larmor-type precession and slow, barrier-crossing dynamics. Here, a diffusion-jump model is proposed to take advantage of the timescale separation and to approximate barrier-crossings as thermally activated jump processes that occur alongside rotational diffusion. The predictions of our diffusion-jump model are compared to reference results obtained by solving the stochastic Landau-Lifshitz equation coupled to rotational Brownian motion. Good agreement is found in the regime of high energy barriers where Néel relaxation can be considered a thermally activated rare event. While many works in the field have neglected Néel relaxation altogether, our approach opens the possibility to efficiently include Néel relaxation also into interacting many-particle models.
\end{abstract}




\section{INTRODUCTION}

Magnetic nanoparticles that are suspended in a non-magnetic carrier fluid (so-called ferrofluids) show interesting phenomena in the presence of external magnetic fields [1]. The magnetoviscous effect, for example, has attracted considerable attention since the effective viscosity depends on an externally applied magnetic field [2]. Over the last years, there has been growing interest also in biomedical applications of magnetic nanoparticles [3, 4].

From a modeling point of view, mesoscopic models of ferrofluids have proven to be very fruitful, where individual magnetic nanoparticles are considered explicitly but the solvent is treated as a viscous medium [2]. In the majority of previous studies, the dynamics of suspended magnetic nanoparticles has been modeled using the rigid-dipole approximation, both, for interacting as well as non-interacting systems, (see e.g. [1, 2, 5-9] and references therein). The rigid-dipole approximation assumes that the magnetic moment is permanently locked within the particle. This approximation is justified when particles are sufficiently large so that the magnetic anisotropy energy is much larger than thermal energy. At the same time, the magnetic core of the particles must be small enough so that it remains a magnetic single domain. In addition, we allow particles to rotate within the suspending viscous medium.

The rigid-dipole approximation breaks down when internal, so-called Néel, relaxation becomes important [10]. Such situations occur e.g. (i) for smaller or magnetically weak particles where the energy barrier for Néel relaxation can be overcome on relevant time scales, or (ii) when particle rotation is severely hindered or nearly suppressed. Particles which fall under category (i) are usually ignored in the modeling since they are either considered irrelevant for viscous properties [11] or are treated as a magnetic background [12]. In both cases, however, internal relaxation needs to be accounted for when considering e.g. magnetorelaxometry or hyperthermia applications.

Models beyond the rigid-dipole approximation have been proposed by Shliomis and Stepanov [13, 14] and by Coffey, Cregg, and Kalmykov [10], where the Brownian rotational diffusion of the particle is combined with the stochastic Landau-Lifshitz equation to describe the internal magnetization dynamics. Emerging biomedical applications such as magnetorelaxometry and hyperthermia have sparked renewed interest in the field $[3,4,15]$. Some authors studied the simplified case of internal magnetization dynamics for a random collection of fixed particle easy axis orientation [16]. Models with combined Brownian and Néel relaxation very similar to that proposed by Shliomis and Stepanov were used in Refs. [17] and [18] to simulate magnetization hysteresis curves in oscillating external fields and magnetization relaxation of suspended multi-core particles, respectively.

While the model proposed by Shliomis and Stepanov is physically sound, its numerical simulation often runs into practical difficulties due to a separation of time scales. For magnetic nanoparticles on the order of $10 \mathrm{~nm}$, the internal time scale for magnetization diffusion in the Landau-Lifshitz equation is typically much smaller than the Brownian and Néel times. In such cases, straightforward simulations of the Shliomis and Stepanov model is not only highly inefficient but practically intractable since the Brownian and Néel relaxation processes can not be simulated with reasonable computational effort. In order to address this challenge, Berkov et al. simulated the fast remagnetization process of interacting magnetic nanoparticles with a hybrid scheme, where the positions and orientations of the particles are integrated with Langevin dynamics, while the magnetic moments are equilibrated using a Monte-Carlo method [19]. The authors also showed the limitations of the rigid-dipole approximation for correctly capturing the magnetization relaxation dynamics [20]. Ruta et al. [21] and Jonasson et al. [22] used kinetic Monte-Carlo schemes to simulate the magnetization response of frozen multi-core particles to oscillating magnetic fields.

Here, our aim is to benefit from the time-scale separation in order to establish a well-founded and more efficient model that is able to approximate the predictions of the Shliomis and Stepanov model over the relevant long time scales. We limit ourselves here to the case of non-interacting particles in order to carefully develop and study this model that extends those in Refs. [20-22] by including barrier crossings and Brownian rotation, respectively, in a physically sound manner. As a first step, we numerically solve the model of Shliomis and Stepanov to obtain reference results for magnetization relaxation, dynamic magnetic susceptibility and magnetoviscosity over a certain parameter range. In order to tackle the problem of time-scale separation, we propose a diffusion-jump model that combines the nonequilibrium Brownian rotational dynamics with jump processes that model the rare, thermally activated magnetization reversals within each particle. By comparing the predictions of the diffusion-jump model to the reference results for the Shliomis and Stepanov model we establish the range of validity of the new model.

The paper is organized as follows. The model of Shliomis and Stepanov is briefly reviewed in Sect. II, where we also give its stochastic formulation. We introduce the new diffusion-jump model in Sect. III. Results of both models are shown and compared in Sect. IV before we offer some conclusions in Sect. V. A number of technical details are given in the appendix. 


\section{MICROSCOPIC "EGG" MODEL}

We here briefly summarize the so-called "egg model" proposed by Shliomis and Stepanov to describe the internal magnetization dynamics coupled to the Brownian rotation of a magnetic nanoparticle. Further details can be found in Ref. [14].

In the egg model, the state of the nanoparticle is described by two vectors: (i) the orientation of the nanoparticle $\mathbf{n}$; (ii) the magnetic moment $m \mathbf{e}$, where the magnitude of the magnetic moment $m$ is constant and given by $m=M_{\mathrm{s}} v_{\mathrm{m}}$. The spontaneous magnetization of the magnetic material is denoted by $M_{\mathrm{s}}$ and $v_{\mathrm{m}}$ is the volume of the magnetic core of the nanoparticle. Both, $\mathbf{e}$ and $\mathbf{n}$ are treated as three-dimensional unit vectors. The resulting magnetization is given by $\mathbf{M}=n m\langle\mathbf{e}\rangle$, where $n$ is the number density of magnetic nanoparticles and $\langle\bullet\rangle$ denotes thermal averages. For point (i) above, we assume the particle is rotationally symmetric so that its orientation can be described by the unit vector $\mathbf{n}$. The implicit assumption made for (ii) is that the magnetic nanoparticle is sufficiently small so that it can be treated as a magnetic mono-domain. For Cobalt and magnetite, for example, this assumption is fulfilled for particle diameters less than approximately 24 and $70 \mathrm{~nm}$, respectively [1]. Since this formulation allows the magnetic moment ("yolk") to rotate inside the particle, the corresponding model is also know as "egg model".

In the following we assume that the nanoparticles are spherical. Generalizations to treat particles which are ellipsoids of revolution can be done in a similar manner as in the chain model of ferrofluids [6, 23]. Furthermore, we assume the nanoparticles have uniaxial anisotropy. Then, we let the orientation of the particle $\mathbf{n}$ coincide with the direction of the easy axis of the magnetic material, so that the energy in an external magnetic field $\mathbf{H}$ is given by

$$
U(\mathbf{e}, \mathbf{n})=-m \mathbf{e} \cdot \mathbf{H}-K v_{\mathrm{m}}(\mathbf{e} \cdot \mathbf{n})^{2}
$$

with $K$ the anisotropy constant of the magnetic material [1]. It is important to emphasize that the egg model disregards interactions between the nanoparticles and is therefore valid only in the ultra-dilute limit. The equilibrium properties of the model are described by the Boltzmann distribution,

$$
F_{\mathrm{eq}}(\mathbf{e}, \mathbf{n})=\frac{1}{Z_{\mathrm{eq}}} \exp \left[\mathbf{e} \cdot \mathbf{h}+\kappa(\mathbf{e} \cdot \mathbf{n})^{2}\right]
$$

and have been discussed in detail in Ref. [1]. In Eq. (2) we introduced the dimensionless magnetic field $\mathbf{h}=m \mathbf{H} / k_{\mathrm{B}} T$, $h=|\mathbf{h}|$ is the Langevin parameter, and the dimensionless anisotropy constant $\kappa=K v_{\mathrm{m}} / k_{\mathrm{B}} T$, where $k_{\mathrm{B}}$ and $T$ denote Boltzmann's constant and temperature, respectively.

In order to couple the internal magnetization dynamics of the nanoparticle to its rotational Brownian motion, Shliomis and Stepanov suggested the following Fokker-Planck equation for the time-dependent joint probability density $F(\mathbf{e}, \mathbf{n} ; t)[14]$ :

$$
\begin{aligned}
\frac{\partial F}{\partial t} & =-\left(\mathcal{L}_{e}+\mathcal{L}_{n}\right) \cdot[\boldsymbol{\Omega} F]-\mathcal{L}_{e} \cdot\left[\boldsymbol{\omega}_{L} F\right] \\
& +\left(\frac{1}{2 \tau_{\mathrm{B}}}\left(\mathcal{L}_{e}+\mathcal{L}_{n}\right) \cdot F\left(\mathcal{L}_{e}+\mathcal{L}_{n}\right)+\frac{1}{2 \tau_{\mathrm{D}}} \mathcal{L}_{e} \cdot F \mathcal{L}_{e}\right)\left[\frac{U}{k_{\mathrm{B}} T}+\ln F\right]
\end{aligned}
$$

with the rotational operators $\mathcal{L}_{e}=\mathbf{e} \times \partial / \partial \mathbf{e}$ and $\mathcal{L}_{n}=\mathbf{n} \times \partial / \partial \mathbf{n}$. The Brownian rotational relaxation time of the particle is defined as $\tau_{\mathrm{B}}=3 \eta_{\mathrm{s}} v_{\mathrm{h}} / k_{\mathrm{B}} T$, where $\eta_{\mathrm{s}}$ is the solvent viscosity and $v_{\mathrm{h}}$ the hydrodynamic volume of the nanoparticle. The second characteristic time in Eq. (3) is the internal rotational diffusion time, $\tau_{\mathrm{D}}=M_{\mathrm{s}} v_{\mathrm{m}} /\left(2 \alpha \gamma k_{\mathrm{B}} T\right)$, with $\alpha$ the dimensionless damping parameter and $\gamma$ the gyromagnetic ratio. The Larmor frequency is defined as and given here by [14]

$$
\boldsymbol{\omega}_{L}=\gamma \mathbf{H}_{\mathrm{eff}}=-\frac{\gamma}{m} \frac{\partial U}{\partial \mathbf{e}}=\mathbf{H}+2 K M_{\mathrm{s}}^{-1}(\mathbf{e} \cdot \mathbf{n}) \mathbf{n} .
$$

For later use, we note that we can rewrite this expression as $\tau_{D} \boldsymbol{\omega}_{L}=\frac{1}{2 \alpha} \mathbf{h}+\frac{\kappa}{\alpha}(\mathbf{e} \cdot \mathbf{n}) \mathbf{n}$. Finally, the vector $\boldsymbol{\Omega}$ denotes one half of the vorticity of an externally applied flow field. By construction, the equilibrium probability density (2) is a stationary solution of Eq. (3) for $\boldsymbol{\Omega}=\mathbf{0}$.

We here also give the stochastic formulation corresponding to the Fokker-Planck equation (3), which provides further insight into the model and serves as a starting point for numerical solutions. Inserting the potential $U$ from Eq. (1) and identifying drift and diffusion coefficients from Eq. (3), we follow standard procedures [24] to formulate the corresponding stochastic differential equations for the random vectors $\mathbf{e}_{t}$ and $\mathbf{n}_{t}$,

$$
\begin{aligned}
\frac{\mathrm{d}}{\mathrm{d} t} \mathbf{e}_{t} & =\left[\boldsymbol{\Omega}+\boldsymbol{\omega}_{L}+\frac{1}{2 \tau_{0}} \mathbf{e}_{t} \times \mathbf{h}+\frac{1}{\tau_{\mathrm{D}}} \kappa\left(\mathbf{e}_{t} \cdot \mathbf{n}_{t}\right) \mathbf{e}_{t} \times \mathbf{n}_{t}\right] \times \mathbf{e}_{t}+\left[\mathbf{b}_{t}^{(e)}+\mathbf{b}_{t}^{(n)}\right] \times \mathbf{e}_{t} \\
\frac{\mathrm{d}}{\mathrm{d} t} \mathbf{n}_{t} & =\left[\boldsymbol{\Omega}+\frac{1}{2 \tau_{\mathrm{B}}}\left(\mathbf{e}_{t} \times \mathbf{h}\right)\right] \times \mathbf{n}_{t}+\mathbf{b}_{t}^{(n)} \times \mathbf{n}_{t},
\end{aligned}
$$


where the Brownian contributions $\mathbf{b}_{t}^{(e)}, \mathbf{b}_{t}^{(n)}$ are independent three-dimensional Gaussian random variables with zero mean, $\left\langle\mathbf{b}_{t}^{(e)}\right\rangle=\left\langle\mathbf{b}_{t}^{(n)}\right\rangle=\mathbf{0}$, and variance $\left\langle\mathbf{b}_{t}^{(e)} \mathbf{b}_{s}^{(e)}\right\rangle=\left(1 / \tau_{\mathrm{D}}\right) \delta(t-s) \mathbf{I},\left\langle\mathbf{b}_{t}^{(n)} \mathbf{b}_{s}^{(n)}\right\rangle=\left(1 / \tau_{\mathrm{B}}\right) \delta(t-s) \mathbf{I},\left\langle\mathbf{b}_{t}^{(e)} \mathbf{b}_{s}^{(n)}\right\rangle=\mathbf{0}$, where $\delta(t)$ denotes the Dirac delta function and $\mathbf{I}$ the three-dimensional unit matrix. In Eq. (5) we defined the effective relaxation time $\tau_{0}$ by

$$
\frac{1}{\tau_{0}}=\frac{1}{\tau_{\mathrm{B}}}+\frac{1}{\tau_{\mathrm{D}}} .
$$

Further details on the derivation of Eqs. (5) and (6) are provided in appendix B.

Some comments seem in order. First, in the rigid-dipole approximation the magnetization direction is always aligned with the easy axis of the particle, $\mathbf{e}_{t}=\mathbf{n}_{t}$, and Eq. (6) reduces to the standard ferrofluid model of non-interacting magnetic nanoparticles [2]. Without fluctuations and for stationary particles, the above equation for e, Eq. (5), reduces to the Landau-Lifshitz equation. Here, since fluctuations are added only on the precession term but not on the relaxation term, the equation reduces for stationary particles to the "stochastic Landau-Lifshitz" equation [25]. When fluctuations are added on precession and relaxation term, the model coincides with the one used in [18] in the low-damping regime appropriate for magnetic nanoparticles. This connection is easily established by relating the quantities $\mathbf{b}_{t}^{(e)}$ to the fluctuating fields $\mathbf{H}_{t}^{\mathrm{f}}=\mathbf{b}_{t}^{(e)} / \gamma$ used in Refs. [18, 25]. The variance of the fluctuating fields is given by $\left\langle\mathbf{H}_{t}^{\mathrm{f}} \mathbf{H}_{s}^{\mathrm{f}}\right\rangle=2 D_{\mathrm{LLG}} \delta(t-s) \mathbf{I}$ with $D_{\mathrm{LLG}}=\alpha k_{\mathrm{B}} T /(\gamma m)$. Note that some authors introduce the renormalized gyromagnetic ratio $\gamma \rightarrow \gamma /\left(1+\alpha^{2}\right)$ so that $D_{\mathrm{LLG}}=\alpha /\left(1+\alpha^{2}\right)\left(k_{\mathrm{B}} T / \gamma m\right)[25]$.

\section{DIFFUSION-JUMP MODEL}

For typical magnetic nanoparticles made of magnetite with diameters of some $10 \mathrm{~nm}$, the basic time scale of internal magnetization diffusion $\tau_{\mathrm{D}}$ in the Landau-Lifshitz equation is on the order of $10^{-10} \ldots 10^{-9} \mathrm{~s}$, while the Brownian and Néel relaxation are typically much slower with $\tau_{\mathrm{N}}, \tau_{\mathrm{B}} \approx 10^{-7} \ldots 10^{-5} \mathrm{~S}$ in standard solvents and ambient temperature $[1,19]$. Although the egg model presented above remains valid in these cases, the condition $\tau_{\mathrm{D}} \ll \tau_{\mathrm{B}}, \tau_{\mathrm{N}}$ implies that the numerical solution of Eqs. (5) and (6) will become very inefficient or even impractical with standard computational resources.

The Néel relaxation time $\tau_{\mathrm{N}}$ increases very strongly with the magnetic volume of the particle and for $\tau_{\mathrm{N}} \rightarrow \infty$ the rigid-dipole approximation becomes exact. But what are the corrections when $\tau_{\mathrm{N}}$ is large but finite? We are interested in the situation $\tau_{\mathrm{N}} \gg \tau_{\mathrm{D}}$ where Néel relaxation becomes a rare event compared with internal diffusion. In this regime, Néel relaxation is a thermally activated process that corresponds to large magnetic anisotropy energies compared to thermal energy, $\kappa \gg 1$ (see Sect. C and Eq. (C22) for a derivation of Brown's classical result of the Néel relaxation time in this regime). Estimates for magnetite particles $\left(K \approx 10^{4} \mathrm{~J} / \mathrm{m}^{3}\right)$ with core diameters of 15 and 20 nm give $\kappa \approx 4$ and $\kappa \approx 10$ at ambient temperatures, respectively. Under such conditions, it is reasonable to assume that the magnetic moment will be well aligned with the easy axis of the magnetic particle, $\mathbf{e} \approx \pm \mathbf{n}$. We therefore model Néel relaxation as a Poisson process with rate $\lambda$ of jumps $\mathbf{e} \leftrightarrows-\mathbf{e}$. For a Poisson process, the probability that no jump has occurred over the time interval $\Delta t$ equals $\exp [-\lambda \Delta t]$, while the probability for exactly one jump in $\Delta t$ equals $1-\exp [-\lambda \Delta t][24]$. It is important to emphasize that the probability of two or more jumps in $\Delta t$ is negligible for Poisson processes. This condition is fulfilled for large enough $\kappa$ and small enough time intervals $\Delta t$. While this requirement for Poisson processes is always met in the cases considered below, it is important to emphasize that the assumption $\mathbf{e} \approx \pm \mathbf{n}$ is much more restrictive and limits the model to the regime of rather large magnetic anisotropy energies compared to thermal energy.

Adopting the approximation $\mathbf{e}= \pm \mathbf{n}$, the particle orientation $\mathbf{n}$ is no longer an independent degree of freedom. Let $f(\mathbf{e} ; t)$ denote the probability density to find the magnetization orientation $\mathbf{e}$ at time $t$. We propose the following diffusion-jump process as a model for the magnetization dynamics

$$
\frac{\partial}{\partial t} f(\mathbf{e} ; t)=\mathrm{L} f(\mathbf{e} ; t)+\int\left[w\left(\mathbf{e} \mid \mathbf{e}^{\prime}\right) f\left(\mathbf{e}^{\prime} ; t\right)-w\left(\mathbf{e}^{\prime} \mid \mathbf{e}\right) f(\mathbf{e} ; t)\right] \mathrm{d} \mathbf{e}^{\prime},
$$

where L denotes the Fokker-Planck operator describing rotational diffusion subject to the external magnetic field and vorticity in the rigid-dipole approximation $[1,2]$. The second term on the right hand side of Eq. (8) models jump processes with transition rates $w$. Here and in the following, $\int \bullet$ de denote integration over the three-dimensional unit sphere. Equations of the type (8) are known as "differential Chapman-Kolmogorov" equations [24].

The above assumption that $\mathbf{e}= \pm \mathbf{n}$ suggests that the transition rates $w$ vanish unless $\mathbf{e}^{\prime}=-\mathbf{e}$. Also, for thermal activation, the rate depends on the energy of the initial state and the barrier, but is independent of the final states. Therefore we approximate the transition rates as

$$
w\left(\mathbf{e}^{\prime} \mid \mathbf{e}\right)=\delta\left(\mathbf{e}^{\prime}+\mathbf{e}\right) \lambda(\mathbf{e})
$$


so that Eq. (8) simplifies to

$$
\frac{\partial}{\partial t} f(\mathbf{e} ; t)=\mathrm{L} f(\mathbf{e} ; t)+[\lambda(-\mathbf{e}) f(-\mathbf{e} ; t)-\lambda(\mathbf{e}) f(\mathbf{e} ; t)]
$$

We note that Eqs. (8) and (10) conserve the normalization of the probability density $\int f(\mathbf{e} ; t)$ de $=1$.

Next, we require the Boltzmann equilibrium $f_{\text {eq }}(\mathbf{e})=\exp [\mathbf{e} \cdot \mathbf{h}] / z_{\text {eq }}$ to be a stationary solution to the differential Chapman-Kolmogorov equation in the absence of flow, $\boldsymbol{\Omega}=\mathbf{0}$. Since $\mathrm{L} f_{\mathrm{eq}}=0$, this requirement is met when the jump rates satisfy the detailed balance condition

$$
\frac{\lambda(-\mathbf{e})}{\lambda(\mathbf{e})}=\frac{f_{\mathrm{eq}}(\mathbf{e})}{f_{\mathrm{eq}}(-\mathbf{e})}=\exp [2 \mathbf{e} \cdot \mathbf{h}]
$$

We use the ansatz $\lambda(\mathbf{e})=\lambda_{0} r(|\mathbf{e} \cdot \mathbf{h}|) \exp [-\mathbf{e} \cdot \mathbf{h}]$ which satisfies Eq. (11) identically for arbitrary rate factors $r$. Due to its interpretation as a transition rate, the dimensionless factor $r$ should be non-negative, $r(x) \geq 0$. We also require $r(0)=1$ so that $\lambda_{0}$ denotes the transition rate in the absence of an external field. As shown below, the identification $\lambda_{0}=1 /\left(2 \tau_{\mathrm{N}}\right)$ leads to a correct description of the Néel relaxation on the time scale $\tau_{\mathrm{N}}$. Note that $\lambda_{0}$ can be identified with the probability flux $\bar{J}_{y}$, Eq. (C21), across the anisotropy barrier.

With the explicit form of the operator L, we thus propose the following diffusion-jump process to add internal Néel relaxation to the rotational Brownian motion of magnetic nanoparticles in the presence of external fields and flow,

$$
\begin{aligned}
\frac{\partial}{\partial t} f(\mathbf{e} ; t) & =-\mathcal{L}_{e} \cdot\left[\left(\boldsymbol{\Omega}+\frac{1}{2 \tau_{\mathrm{B}}} \mathbf{e} \times \mathbf{h}\right) f(\mathbf{e} ; t)\right]+\frac{1}{2 \tau_{\mathrm{B}}} \mathcal{L}_{e}^{2} f(\mathbf{e} ; t) \\
& +\frac{r(|\mathbf{e} \cdot \mathbf{h}|)}{2 \tau_{\mathrm{N}}}\left[e^{\mathbf{e} \cdot \mathbf{h}} f(-\mathbf{e} ; t)-e^{-\mathbf{e} \cdot \mathbf{h}} f(\mathbf{e} ; t)\right]
\end{aligned}
$$

In the limit $\tau_{\mathrm{N}} \rightarrow \infty$, the jump processes described by the last term on the right hand side of Eq. (12) vanish and the model reduces to the classical model within the rigid-dipole approximation. For finite values of $\tau_{\mathrm{N}}$, the model goes beyond the rigid-dipole approximation by including thermally activated magnetization reversals. When rotational motion of the particle is suppressed, $\tau_{\mathrm{B}} \rightarrow \infty$ and $\boldsymbol{\Omega}=\mathbf{0}$, internal magnetization reversals become the only relaxation mode. It is important to emphasize that we expect the model (12) to be a valid approximation to the egg model (3) for $\kappa \gg 1$. Otherwise, the assumption of perfect alignment of magnetization and easy axis and of a thermally activated jump process are not justified.

By construction, the diffusion-jump model (12) conserves the normalization of probability, $\int f(\mathbf{e} ; t)$ de $=1$, and has $f_{\text {eq }}$ as a stationary solution for $\boldsymbol{\Omega}=\mathbf{0}$. Furthermore, we can derive the time evolution equation for the reduced magnetization from the differential Chapman-Kolmogorov equation (12) in a standard manner. Let $\langle\mathbf{e}\rangle_{t}=\int \mathbf{e} f(\mathbf{e} ; t)$ de denote the mean magnetization direction at time $t$. Then $\frac{\mathrm{d}}{\mathrm{d} t}\langle\mathbf{e}\rangle_{t}=\int \mathbf{e} \frac{\partial}{\partial t} f(\mathbf{e} ; t)$ de and upon inserting the right hand side of Eq. (12) for $\frac{\partial}{\partial t} f$ we arrive at the equation for the expectation value of the reduced magnetization,

$$
\frac{\mathrm{d}}{\mathrm{d} t}\langle\mathbf{e}\rangle_{t}=\left\langle\mathrm{L}^{\dagger} \mathbf{e}\right\rangle_{t}+\frac{1}{2 \tau_{\mathrm{N}}}\left[\int r(|\mathbf{e} \cdot \mathbf{h}|) e^{\mathbf{e} \cdot \mathbf{h}} \mathbf{e} f(-\mathbf{e} ; t) \mathrm{d} \mathbf{e}-\left\langle r(|\mathbf{e} \cdot \mathbf{h}|) e^{-\mathbf{e} \cdot \mathbf{h}} \mathbf{e}\right\rangle_{t}\right],
$$

where $\mathrm{L}^{\dagger}$ denotes the transpose of the Fokker-Planck operator defined above. The term $\left\langle\mathrm{L}^{\dagger} \mathbf{e}\right\rangle_{t}$ appears identically in the rigid-dipole model and can be taken over from Ref. [5] (the calculations can also be found in other references, like e.g. [26]). The term proportional to $\tau_{\mathrm{N}}^{-1}$ is new and models Néel relaxation. With the change of integration variable $\mathbf{e} \rightarrow \mathbf{e}^{\prime}=-\mathbf{e}$ and noting that the Jacobian of this transformation is one, we find that both terms in the square bracket add up to $-\tau_{\mathrm{N}}^{-1}\left\langle r e^{-\mathbf{e} \cdot \mathbf{h}} \mathbf{e}\right\rangle_{t}$. Putting these results together gives the magnetization equation

$$
\frac{\mathrm{d}}{\mathrm{d} t}\langle\mathbf{e}\rangle_{t}=\boldsymbol{\Omega} \times\langle\mathbf{e}\rangle_{t}+\frac{1}{2 \tau_{\mathrm{B}}}\left(\mathbf{h}-\langle\mathbf{e e}\rangle_{t} \cdot \mathbf{h}\right)-\frac{1}{\tau_{\mathrm{B}}}\langle\mathbf{e}\rangle_{t}-\frac{1}{\tau_{\mathrm{N}}}\left\langle r(|\mathbf{e} \cdot \mathbf{h}|) e^{-\mathbf{e} \cdot \mathbf{h}} \mathbf{e}\right\rangle_{t} .
$$

The first three terms on the right hand side of Eq. (12) correspond to the rotation with the flow vorticity, the external magnetic field and Brownian relaxation, respectively, and appear identically in the rigid-dipole model [2, 5]. The last term proportional to $\tau_{\mathrm{N}}^{-1}$ is new and describes Néel relaxation. In the absence of an external field, $\mathbf{h}=\mathbf{0}$, this term simplifies to $-\tau_{\mathrm{N}}^{-1}\langle\mathbf{e}\rangle_{t}$ as it should. In the presence of an external field, the Néel term in general depends on the particular form of the rate factor $r$. In equilibrium, however, it is readily verified that this term vanishes, $\left\langle r e^{-\mathbf{e} \cdot \mathbf{h}} \mathbf{e}\right\rangle_{\text {eq }}=\mathbf{0}$, due to symmetry, irrespective of the choice of $r$. Therefore, as expected, the additional Néel relaxation term does not change the equilibrium magnetization. In the following, we study properties of this model and explore its range of validity by comparison to simulations of the egg model of Sect. II. 
In order to fully specify the model, we need to fix the modification of the transition rate due to the externally applied field described by $r$. While the calculation of $\lambda_{0}$ from the model in Sect. II is standard, the corresponding result in the presence of an external field is unfortunately not available (see also appendix C), to the best of our knowledge. We show below that the magnetization relaxation and magnetic susceptibility are independent of the particular choice of $r$. But in order to make further progress for calculating the rotational viscosity, we will use in Sect. IV C the phenomenological expression $r(x)=\cosh (a x)$ with parameter $a$ in the range $0 \leq a \leq 1$. Then, the transition rates can be expressed as $\lambda(\mathbf{e})=\left(4 \tau_{\mathrm{N}}\right)^{-1}[\exp (-(1-a) \mathbf{e} \cdot \mathbf{h})+\exp (-(1+a) \mathbf{e} \cdot \mathbf{h})]$. For $a=0, \lambda$ simplifies to an Arrhenius-like expression. Note that the "Glauber"-like choice $r(x)=\operatorname{sech}(x)$ leading to $\lambda(\mathbf{e})=\left(2 \tau_{\mathrm{N}}\right)^{-1}[1+\tanh (-\mathbf{e} \cdot \mathbf{h})]$ also satisfies detailed balance and therefore is equally admissible. For the present case, both choices give very similar results for $a=0$ (see the discussion in the appendix D).

\section{RESULTS}

\section{A. Magnetization relaxation}

Probing the relaxation of the magnetization after switching off a strong ordering field is not only a common method to study the orientational dynamics, but is also used as a diagnostic tool in biomedicine [3]. Here, we study the magnetization relaxation by starting all simulations in a perfectly oriented initial state and follow the orientational dynamics in the absence of external fields, $\mathbf{h}=\boldsymbol{\Omega}=\mathbf{0}$. We use a Heun algorithm to integrate Eqs. (5), (6) numerically with an ensemble of $10^{5}$ independent realizations. The time step is chosen as $2 \times 10^{-3} \tau_{\mathrm{D}}$.

For the egg model, we find the short time behavior $\langle\mathbf{e}\rangle_{t}=1-t / \tau_{0}+\mathcal{O}\left(t^{2}\right)$. Therefore, the initial magnetization decay is determined by $\tau_{0}$. For longer times, we expect a different behavior due to Brownian and Néel relaxation. In fact, for the diffusion-jump model we find from Eq. (14) for the magnetization relaxation $\frac{\mathrm{d}}{\mathrm{d} t}\langle\mathbf{e}\rangle_{t}=-\tau_{\text {eff }}^{-1}\langle\mathbf{e}\rangle_{t}$, i.e. a single-exponential decay with the effective relaxation time $\tau_{\text {eff }}$ given by

$$
\frac{1}{\tau_{\text {eff }}}=\frac{1}{\tau_{\mathrm{B}}}+\frac{1}{\tau_{\mathrm{N}}}
$$

Note that contrary to $\tau_{0}$ defined in Eq. $(7), \tau_{\text {eff }}$ is defined as the effective relaxation time resulting from Brownian and Néel relaxation. Since we are considering magnetization relaxation in the absence of external fields, this result holds for all admissible choices of rate factors $r$.

In Fig. 1, the magnetization relaxation is shown for different values of the anisotropy constant $\kappa$. Except for a short-time initial transient of order $\tau_{0}$, we find that the relaxation can be very well described by a single-exponential decay with effective relaxation time $\tau_{\text {eff }}$ for all values of $\kappa$ and $\tau_{\mathrm{B}}$ investigated.
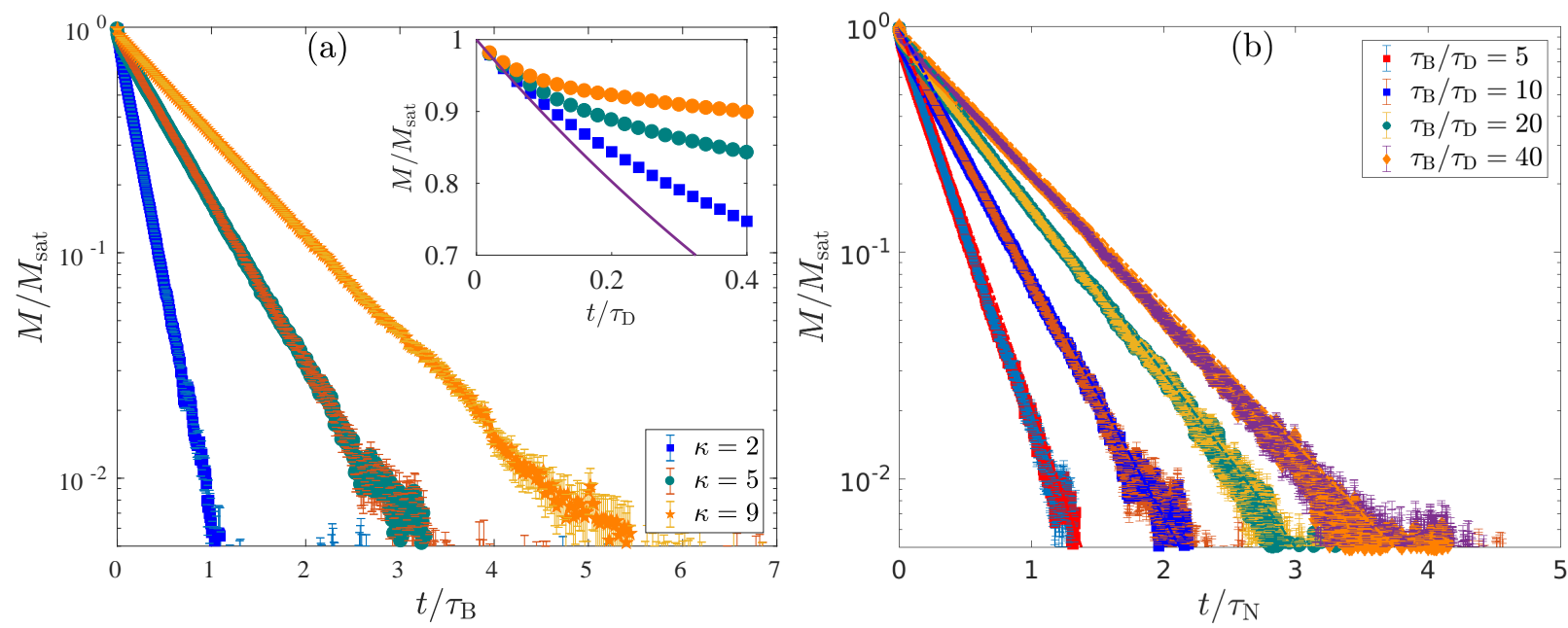

FIG. 1. Magnetization relaxation from perfectly oriented initial state in the absence of external fields is shown on a semilogarithmic plot. Left panel shows the relaxation versus scaled time $t / \tau_{\mathrm{B}}$ where $\tau_{\mathrm{B}} / \tau_{\mathrm{D}}=10$ and different values of $\kappa$ were chosen. The inset is a zoom into the initial relaxation for times smaller than $\tau_{\mathrm{D}}$. The right panel shows the magnetization relaxation when $\kappa=5$ is fixed, time is scaled with $\tau_{\mathrm{N}}$ and different ratios $\tau_{\mathrm{B}} / \tau_{\mathrm{D}}$ are considered.

The effective relaxation times $\tau_{\text {eff }}$ are extracted from a single-exponential fit to the magnetization decay. We only consider times $t>\tau_{\mathrm{D}}$ in order to exclude the fast initial transient. The resulting relaxation times are shown in 
Fig. 2. As $\kappa$ is increased, $\tau_{\text {eff }}$ approaches $\tau_{\mathrm{B}}$ as expected from Eq. (15) since the corresponding Néel relaxation time $\tau_{\mathrm{N}}$ becomes much longer than $\tau_{\mathrm{B}}$. For not too small values of $\kappa$, the approximation $\tau_{\mathrm{N}} \approx \tau_{\mathrm{D}} \sqrt{\pi} e^{\kappa} /\left(2 \kappa^{3 / 2}\right)$ derived by Brown (and re-derived in the present context in appendix C) is quite accurate. The resulting effective relaxation times are shown in Fig. 2 and approximate the numerical results from the magnetization decay quite accurately.

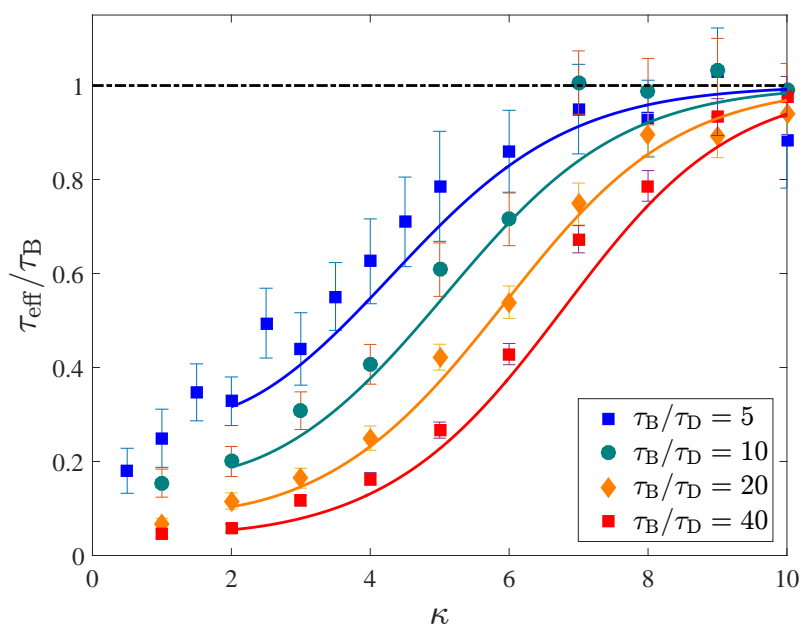

FIG. 2. Effective magnetization relaxation times $\tau_{\text {eff }}$ as a function of $\kappa$ for different values of $\tau_{\mathrm{B}} / \tau_{\mathrm{D}}$. Symbols correspond to numerical results from exponential fits to relaxation curves shown in Fig. 1, while lines correspond to the expected value from Eq. (15) with $\tau_{\mathrm{N}}$ calculated from Brown's expression.

\section{B. Magnetic susceptibility}

The response of suspended magnetic nanoparticles to externally applied magnetic fields does not only provide insight into the system (e.g. concentration of magnetic material, relaxation modes), but is also important for applications such as hyperthermia [27, 28].

In order to calculate the magnetic susceptibility within the diffusion-jump model of Sect. III, we consider only the first order magnetization response to weak, time-dependent external fields $|\mathbf{h}(t)| \ll 1$. Thus, to first order in $h$ we obtain $\langle\mathbf{e e}\rangle_{t} \cdot \mathbf{h}(t) \approx\langle\mathbf{e e}\rangle_{\mathrm{eq}} \cdot \mathbf{h}(t)=(1 / 3) \mathbf{h}(t)$. Furthermore, $\left\langle r(\mathbf{e} \cdot \mathbf{h}) e^{-\mathbf{e} \cdot \mathbf{h}} \mathbf{e}\right\rangle_{t} \approx\langle(1-\mathbf{e} \cdot \mathbf{h}) \mathbf{e}\rangle_{t}=\langle\mathbf{e}\rangle_{t}-(1 / 3) \mathbf{h}(t)$. Note that we have assumed $r(x)=1+\mathcal{O}\left(x^{2}\right)$ for $x \rightarrow 0$ which is consistent with the requirement $r(-x)=r(x)$. Consequently, the magnetic susceptibility is independent of the particular form of $r$. In the absence of external flow, $\boldsymbol{\Omega}=\mathbf{0}$, and with the above results we obtain

$$
\frac{\mathrm{d}}{\mathrm{d} t}\langle\mathbf{e}\rangle_{t}=-\frac{1}{\tau_{\text {eff }}}\langle\mathbf{e}\rangle_{t}+\frac{1}{3 \tau_{\text {eff }}} \mathbf{h}(t)
$$

with the effective relaxation time $\tau_{\text {eff }}$ defined in Eq. (15). For given time-dependent field $\mathbf{h}(t)=m \mathbf{H}(t) / k_{\mathrm{B}} T$, Eq. (16) can be solved to find the induced magnetization $\mathbf{M}(t)=n m\langle\mathbf{e}\rangle_{t}$. For weak, time-dependent external fields $\mathbf{H}(t)$, the induced magnetization can be expressed in terms of the dynamic magnetic susceptibility $\chi(t)$ as $\mathbf{M}(t)=\int_{0}^{t} \chi\left(t-t^{\prime}\right) \mathbf{H}\left(t^{\prime}\right) \mathrm{d} t^{\prime}$. For sinusoidally varying external fields $\mathbf{H}(t)=\mathbf{H}_{0} \sin (\omega t)$ and $\left|\mathbf{H}_{0}\right|$ small enough, the induced magnetization is proportional to $\mathbf{H}_{0}$ and varies with the same frequency $\omega$,

$$
\mathbf{M}(t)=\left[\chi^{\prime}(\omega) \sin (\omega t)-\chi^{\prime \prime}(\omega) \cos (\omega t)\right] \mathbf{H}_{0} .
$$

Equation (17) defines the storage and loss part of the dynamic susceptibility, $\chi^{\prime}$ and $\chi^{\prime \prime}$, respectively. In terms of reduced units, Eq. (17) can be rewritten as $\langle\mathbf{e}\rangle_{t}=\chi_{0}^{-1}\left[\chi^{\prime}(\omega) \sin (\omega t)-\chi^{\prime \prime}(\omega) \cos (\omega t)\right] \mathbf{h}_{0}$. From Eq. (16) we find that the diffusion-jump model predicts a Debye law for the dynamic magnetic susceptibility with effective relaxation time $\tau_{\text {eff }}$,

$$
\chi_{\mathrm{D}}^{\prime}(\omega)=\frac{\chi_{0}}{1+\left(\tau_{\mathrm{eff}} \omega\right)^{2}}, \quad \chi_{\mathrm{D}}^{\prime \prime}(\omega)=\frac{\chi_{0} \tau_{\mathrm{eff}} \omega}{1+\left(\tau_{\mathrm{eff}} \omega\right)^{2}},
$$

where $\chi_{0}=n m^{2} /\left(3 k_{\mathrm{B}} T\right)$ denotes the Langevin susceptibility. For the egg model of Sect. II, Shliomis and Stepanov derived in Ref. [14] the approximate expression $3 \chi^{*}=2 \chi_{\perp}+\chi_{\|} /\left(1+i \omega \tau_{\text {eff }}\right)$ with $\chi_{\perp}=\chi_{0}\left(1-S_{2}\right), \chi_{\|}=\chi_{0}\left(1+2 S_{2}\right)$, 
for the complex susceptibility for not too high frequencies. See appendix A for the definition of $S_{2}$. For large values of the anisotropy constant $\kappa$ where $S_{2} \rightarrow 1$, their expression agrees with Eq. (18).

Thanks to the fluctuation-dissipation theorem, the susceptibility can in principle be obtained from the Fourier transformation of the magnetization relaxation studied in Sect. IV A. For the diffusion-jump model, we obtain the same expressions (16), (18) via this route. For the numerical evaluation of $\chi^{\prime}, \chi^{\prime \prime}$ predicted by the egg model, we here prefer to use Eq. (17) and simulate directly the magnetization response to an oscillating external field for a range of frequencies. In order to ensure that the external field is weak enough so that the system remains in the linear response regime, we require $\left|\mathbf{h}_{0}\right|=\left|m \mathbf{H}_{0} / k_{\mathrm{B}} T\right| \ll 1$. For the present case, we found that simulations with $\left|\mathbf{h}_{0}\right|=0.2$ and 0.5 gave results that are indistinguishable within our numerical accuracy and therefore have used $\left|\mathbf{h}_{0}\right|=0.5$ for computational convenience.

Figure 3 shows $\chi^{\prime}$, the real or storage part of the susceptibility, as a function of frequency $\omega$ of the applied field. As the value of $\kappa$ is increased, we observe that $\chi^{\prime}$ approaches the Debye law, Eq. (18). When frequency is scaled with the effective relaxation time $\tau_{\text {eff }}$, reasonable data collapse of $\chi^{\prime}$ is observed for our simulations if $\omega \lesssim \tau_{\text {eff }}^{-1}$ and not too small values of $\kappa$. It is worth to mention that the predictions of the diffusion-jump model are not reliable at high frequencies $\omega \gtrsim 10 \tau_{\mathrm{B}}^{-1}$ even for relatively large values of $\kappa$ since the intermediate plateau is not captured by Eq. (18). Finally, we remark that for low values of $\kappa$, we find that the egg model predicts an undershoot with negative values of $\chi^{\prime}$ at high frequencies. Contrary to the case considered in Ref. [29], here the negative values of $\chi^{\prime}$ are due to the Larmor precession and not due to inertia effects since we consider a non-inertial model.
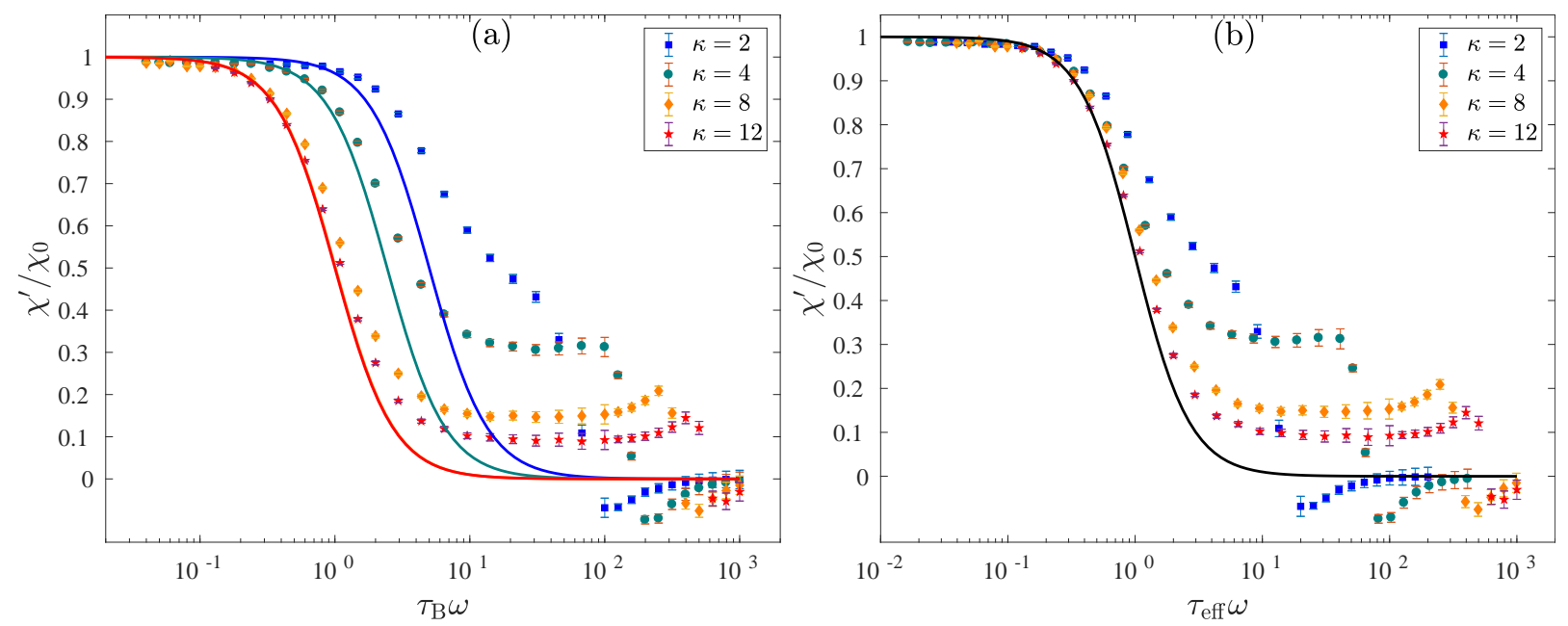

FIG. 3. Scaled real part of the magnetic susceptibility, $\chi^{\prime} / \chi_{0}$, as a function of dimensionless frequency $\omega$ of the applied field. In the left and right panel, the frequency is scaled with the Brownian and effective relaxation time, $\tau_{\mathrm{B}}$ and $\tau_{\text {eff }}$, respectively. Numerical results for different values of $\kappa$ are shown. For the simulations of the egg model, the Brownian relaxation time was chosen as $\tau_{\mathrm{B}}=10 \tau_{\mathrm{D}}$. Solid lines show the Debye law corresponding to Eq. (18).

Figure 4 shows the imaginary or loss part of the susceptibility as a function of frequency of the applied field. As expected, we observe that the position of the main loss peak moves to smaller frequencies as the value of the anisotropy constant $\kappa$ increases. While the position of this peak is rather well described by Eq. (18) due to the increase of $\tau_{\text {eff }}$ with increasing $\kappa$, the model fails to account for the decreasing height of the peak for small values of $\kappa$. The numerical data also show a high frequency peak around the Larmor frequency, which is also not captured by the diffusion-jump model (18). We want to emphasize that the diffusion-jump model is supposed to describe the long-time, small frequency regime and therefore deviations at high frequencies are expected. When the applied frequency is scaled with the effective relaxation time $\tau_{\text {eff }}$, we observe a rather good data collapse for $\omega \lesssim 10 \tau_{\text {eff }}^{-1}$ that approaches the Debye law with increasing $\kappa$.

\section{Magnetoviscosity}

The increase of the effective viscosity of a suspension of magnetic nanoparticles due to an externally applied magnetic field is known as the magnetoviscous effect $[1,2,7]$. From the balance of viscous torques and the torques exerted by 

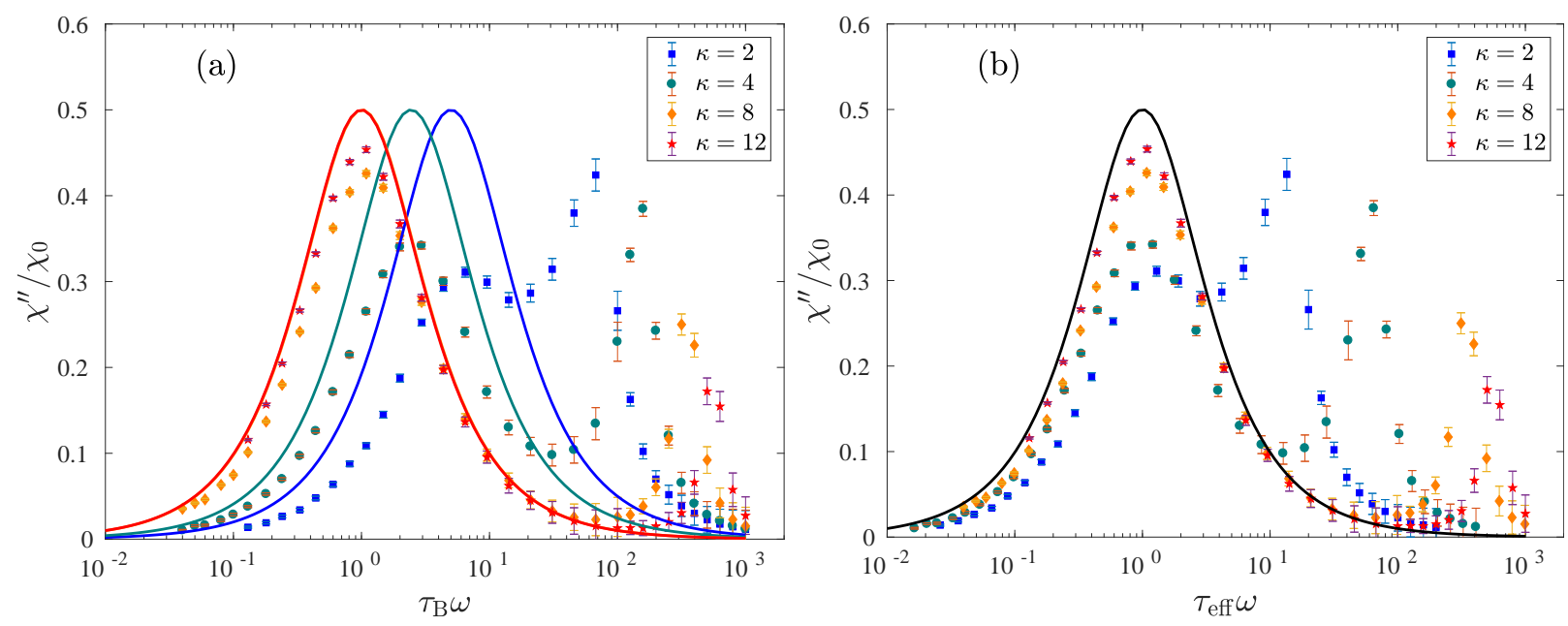

FIG. 4. Same as Fig. 3 but for the imaginary part of the magnetic susceptibility, $\chi^{\prime \prime}$.

the externally applied magnetic field with magnitude $H$, the rotational viscosity can be defined as $[1,2]$

$$
\eta_{\mathrm{rot}}=\frac{M_{\perp} H}{4 \Omega},
$$

where $M_{\perp}$ denotes the magnetization component perpendicular to the applied field. In viscometric flows, the magnitude of the vorticity can be expressed as $\Omega=\dot{\gamma} / 2$ where $\dot{\gamma}$ is the applied shear rate. With the perpendicular magnetization $M_{\perp}=n m\left\langle e^{\perp}\right\rangle$, we can express Eq. (19) also as $\eta_{\text {rot }}=\frac{3}{2} \eta_{\mathrm{s}} \phi\left\langle e^{\perp}\right\rangle h /\left(\tau_{\mathrm{B}} \dot{\gamma}\right)$, where $\phi=n v_{\mathrm{h}}$ is the hydrodynamic volume fraction and $\eta_{\mathrm{s}}$ the viscosity of the solvent.

Within the rigid-dipole approximation, the rotational viscosity becomes $\eta_{\mathrm{rot}}^{\mathrm{Sh}}=(3 / 2) \eta_{\mathrm{s}} \phi h L_{1}^{2}(h) /\left[h-L_{1}(h)\right]$, where $L_{1}(h)=\operatorname{coth}(h)-1 / h$ denotes the Langevin function [5]. Taking into account thermally activated Néel relaxation processes, the value of the rotational viscosity decreases. For the egg model in Sect. II, approximate expressions have been derived in Refs. [1, 13, 30],

$$
\eta_{\mathrm{rot}}^{\mathrm{St}}=\frac{3}{2} \eta_{\mathrm{s}} \phi \times\left\{\begin{array}{cl}
\frac{h^{2}}{18}\left(1+2 S_{2}\right) \frac{\tau_{\text {eff }}}{\tau_{\mathrm{B}}} & \text { for } h \ll 1 \\
\frac{35 L_{2}^{2} S_{2}^{2}}{14+5 L_{2} S_{2}+16 L_{4} S_{4}} & \text { for } h>\kappa
\end{array}\right.
$$

where $L_{n}(h)=\left\langle P_{n}(\mathbf{e} \cdot \hat{\mathbf{h}})\right\rangle_{\text {eq }}$ and $P_{n}(x)$ are Legendre polynomials. The quantities $S_{2}, S_{4}$ are defined in appendix A. Equations (20) correspond to the experimentally relevant case $\tau_{\mathrm{D}} \ll \tau_{\mathrm{B}}$. Some of the limitations of Eqs. (20) have been overcome in Ref. [31] where the expression

$$
\eta_{\mathrm{rot}}^{\mathrm{MSZ}}=\eta_{\mathrm{rot}}^{\mathrm{sat}} \frac{\left[3+2 \kappa S_{2}\right] h L_{1}^{2}(h)}{3 h+2 \kappa S_{2}\left[2+h L_{1}(h)\right] L_{1}(h)}
$$

for the rotational viscosity was derived. The authors of Ref. [31] point out that the value at saturation

$$
\eta_{\mathrm{rot}}^{\mathrm{sat}}=\frac{3}{2} \eta_{\mathrm{s}} \phi \frac{2 \kappa S_{2}}{3+2 \kappa S_{2}}
$$

is reduced due to finite magnetic anisotropy and recovers the rigid-dipole limit $\eta_{\mathrm{rot}}^{\text {sat }} \rightarrow \frac{3}{2} \eta_{\mathbf{s}} \phi$ for $\kappa \rightarrow \infty$.

Within the diffusion-jump model, the rotational viscosity is found to be given by

$$
\eta_{\mathrm{rot}}^{\mathrm{DJ}}=\eta_{\mathrm{rot}}^{\mathrm{sat}} \frac{h L_{1}^{2}(h)}{h-L_{1}(h)}\left(1+\frac{\tau_{\mathrm{B}}}{\tau_{\mathrm{N}}} \ell(h)\right)^{-1}
$$

where $\ell(h)$ is a deceasing function of $h$ such that $\ell(0)=1$ and $\ell \rightarrow 0$ for $h \rightarrow \infty$. In the rigid-dipole limit $\tau_{\mathrm{N}} / \tau_{\mathrm{B}} \rightarrow \infty$, $\eta_{\text {rot }}^{\mathrm{DJ}}$ reduces to $\eta_{\mathrm{rot}}^{\mathrm{Sh}}$ as it should. It is only for intermediate values of $h$ that the detailed form of the function $\ell(h)$ becomes important. The derivation of the rotational viscosity for the diffusion-jump model and the resulting form of $\ell(h)$ are given in appendix D. 
Figure 5 shows the rotational viscosity $\eta_{\text {rot }}$ as a function of the Langevin parameter $h$. The solid black line shows the rigid-dipole limit $\kappa \rightarrow \infty$. We observe that finite values of $\kappa$ lead to a substantial decrease of $\eta_{\text {rot }}$ compared to the rigid-dipole limit. Furthermore, the quadratic approximation (20) is found to be well obeyed for weak enough fields $h \lesssim 0.5$. For larger fields, the second approximation in Eq. (20) is found to underpredict the simulation results, with the notable exception of small anisotropy constants $\kappa=2$ where a good agreement is found (not shown). In the right panel of Fig. 5, the numerical results obtained for the egg model are compared to the diffusion-jump model (23). For the comparison, we choose the rate factor $r$ in the form $r=\cosh (\mathbf{e} \cdot \mathbf{h})$ so that $\ell(h)=\ell_{1}(h)$ is given by Eq. (D9) for $a=1$ (see appendix $\mathrm{D}$ for further details). With this choice of $r$, we observe that the diffusion-jump model reproduces the rotational viscosity of the egg model rather well for weak and strong fields, with some notable discrepancies for intermediate values of $h$.
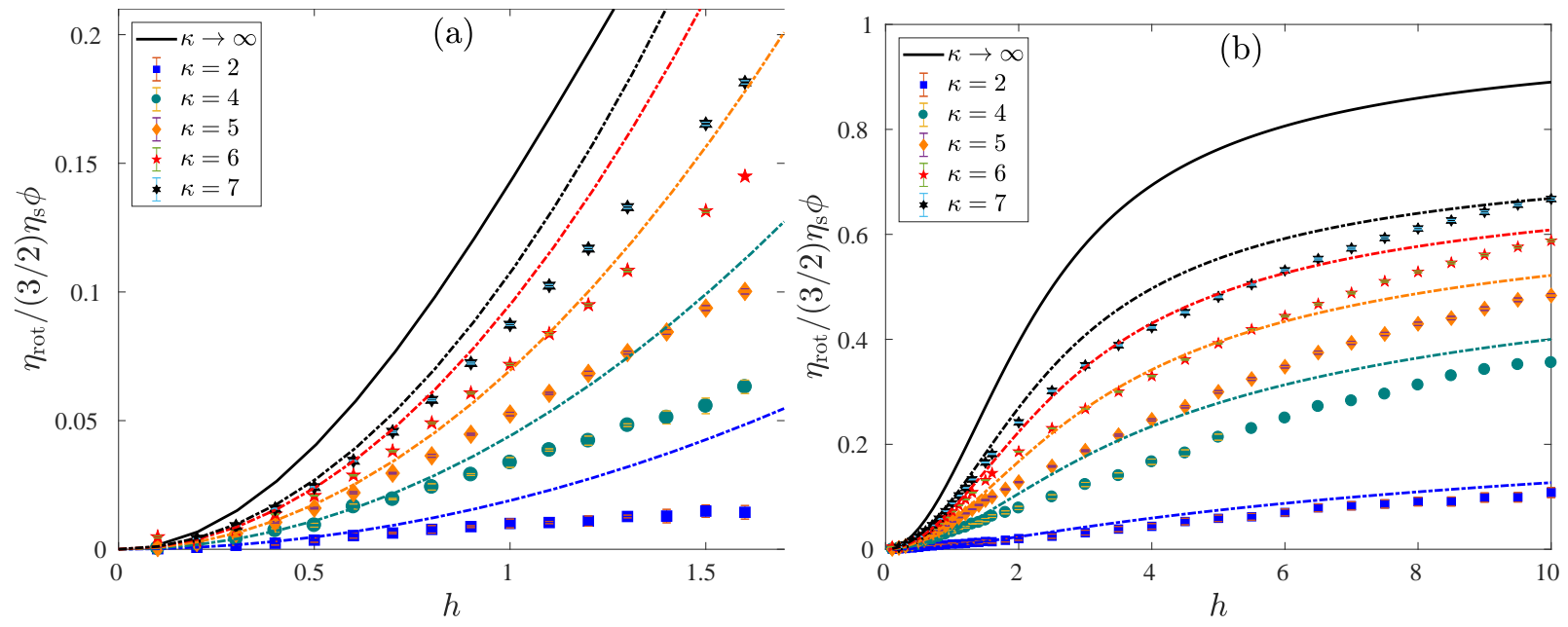

FIG. 5. The scaled rotational viscosity as a function of the Langevin parameter of the applied external field for different values of the dimensionless magnetic anisotropy constant $\kappa$. The left panel shows weak external fields with symbols showing numerical results for the egg model and lines the first of Eqs. (20). The right panel shows the same numerical data but for a larger range of Langevin parameters. The lines here correspond to diffusion-jump model, Eq. (23), for a special form of the rate factor $r$ (see text).

While Eqs. (23) and (20) refer to the important case of weak shear rates, it is well-known that ferrofluids also exhibit viscoelastic effects $[2,32,33]$. In the rigid-dipole approximation, the expression $\eta_{\mathrm{rot}}^{\mathrm{Sh}}=(1 / 4) \eta_{\mathrm{s}} \phi h^{2}\left[1+\left(\tau_{\mathrm{B}} \Omega\right)^{2}\right]^{-1}$ for $h \ll 1$ was derived in Ref. [26] to describe the decrease in viscosity with increasing flow rate. In Fig. 6 , the rotational viscosity is shown as a function of applied shear rate. A Newtonian plateau is reached at low shear rates for all parameter values investigated. We find that finite values of the anisotropy parameter $\kappa$ not only reduce the value of the Newtonian plateau of the rotational viscosity compared to the rigid-dipole case, also the onset of shear thinning moves to higher shear rates. We also observe that the data for $\kappa=8$ are already quite close to the analytical result for rigid dipoles.

\section{CONCLUSIONS}

Here, we study the effect of finite magnetic anisotropy on the magnetization relaxation, dynamic magnetic susceptibility and rotational viscosity of suspended magnetic nanoparticles. Overall, strong deviations from the frequently used rigid-dipole model are observed. Approximate analytical formulae that have been proposed in the literature are found to have a limited range of applicability.

In view of the time-scale gap $\tau_{\mathrm{D}} \ll \tau_{\mathrm{B}}, \tau_{\mathrm{N}}$, we here propose an improvement on the rigid-dipole approximation in terms of a diffusion-jump model that includes thermally activated magnetization reversals. We assume that the magnetization direction coincides with the easy axis of the particle and that the magnetization reversals can be modeled as instantaneous jumps according to a Poisson process. From its construction, the model is expected to be valid for high magnetic anisotropies $\kappa$ and on time scales $t \gtrsim \tau_{\mathrm{B}}, \tau_{\mathrm{N}}$. On these time sales, the magnetization relaxation is to an excellent approximation single-exponential and therefore very well described by the model. Furthermore, the dynamic magnetic susceptibility is also reasonably well described by the diffusion-jump model for low enough frequencies and large enough values of $\kappa$. The diffusion-jump model is able to describe the magnetoviscosity well for weak and strong 


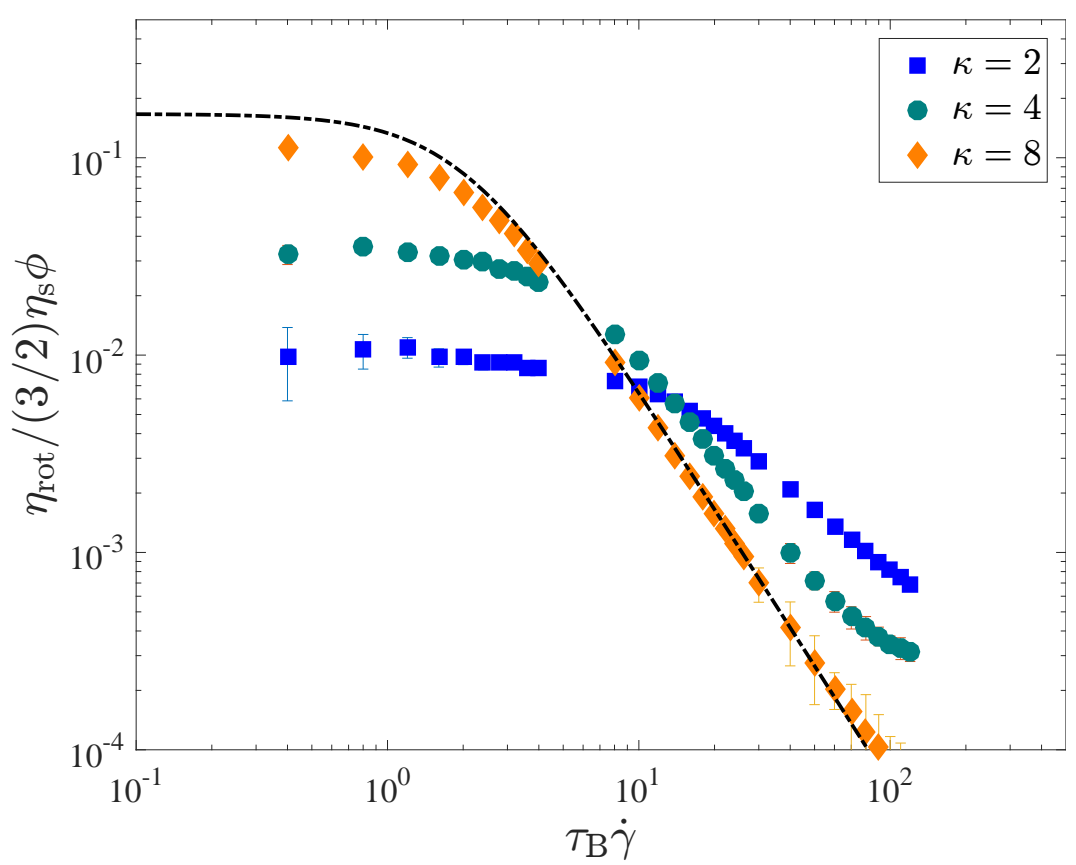

FIG. 6. The rotational viscosity as a function of dimensionless shear rate for different values of the dimensionless magnetic anisotropy constant $\kappa$. The Brownian relaxation time was chosen as $\tau_{\mathrm{B}} / \tau_{\mathrm{D}}=10$ and a moderate magnetic field of strength $h=1$ was applied. The dashed line is the analytical result for the rigid-dipole approximation in weak magnetic fields.

external fields, with some discrepancies at intermediate field strengths. Since we have proposed the field-dependence of the jump rates ad hoc, it is plausible that the prediction of the diffusion-jump model (12) can be improved by choosing a different functional form of $r(|\mathbf{e} \cdot \mathbf{h}|)$ which describes how the external fields modifies the jump rates. Much more satisfying would be a systematic derivation of the field-dependence of the transition rates. Very recently, coarse-graining approaches have been proposed [34] that could be used to address this issue.

In the present work, we have considered the case of non-interacting magnetic nanoparticles in order to develop and test the diffusion-jump model. From a theoretical point of view as well as for a number of practical applications, interactions between magnetic nanoparticles are of great interest. Theoretical and simulation studies building on the rigid-dipole approximation have shown that steric and dipolar interactions can significantly modify the susceptibility and magnetoviscosity [2, 7, 9, 35-37]. The diffusion-jump model proposed here offers the possibility to extend these works to efficiently include Néel relaxation by incorporating magnetization reversals as a Poisson jump process satisfying the detailed balance condition (11). For weakly interacting particles and in the absence of an external field, for example, the rates can be approximated by $\lambda \approx \lambda_{0}$ and therefore the Poisson processes are independent. In general, the interaction of dipolar particles lead to a coupling of the Poisson processes via the local magnetic field-dependence of $\lambda$.

\section{ACKNOWLEDGMENTS}

Valuable discussions with Prof. Quentin Pankhurst, University College London and Dr. Tobias Kuna, University of Reading are gratefully acknowledged. I am also very grateful to Prof. Siegfried Hess who shared with me initial ideas for a jump model already many years ago.

\section{Appendix A: Equilibrium alignment of magnetization and easy axis}

We can ask how well the direction of the easy axis $\mathbf{n}$ and the magnetization e are aligned with each other under equilibrium conditions. The alignment can be quantified in terms of the order parameter $S_{2}=\left\langle P_{2}(\mathbf{e} \cdot \mathbf{n})\right\rangle_{\text {eq }}$ with $P_{2}(x)=\left(3 x^{2}-1\right) / 2$ the second Legendre polynomial and the averages are performed with $F_{\text {eq }}[1]$. From the equilibrium 
Boltzmann distribution (2) we find

$$
\begin{aligned}
S_{2} & =\frac{1}{Z_{\mathrm{eq}}} \int e^{\mathbf{e} \cdot \mathbf{h}} \int\left(\frac{3}{2}(\mathbf{e} \cdot \mathbf{n})^{2}-\frac{1}{2}\right) e^{\kappa(\mathbf{e} \cdot \mathbf{n})^{2}} \mathrm{~d} \mathbf{n} \mathrm{d} \mathbf{e} \\
& =\frac{\int_{0}^{1}\left(\frac{3}{2}(\mathbf{e} \cdot \mathbf{n})^{2}-\frac{1}{2}\right) e^{\kappa z^{2}} \mathrm{~d} z}{\int_{0}^{1} e^{\kappa z^{2}} \mathrm{~d} z} \\
& =\frac{3}{4 \sqrt{\kappa} \mathrm{Da}(\sqrt{\kappa})}-\frac{3}{4 \kappa}-\frac{1}{2}
\end{aligned}
$$

where $\operatorname{Da}(x)=e^{-x^{2}} \int_{0}^{x} e^{y^{2}} \mathrm{~d} y$ denotes the Dawson integral. Comparison of this analytical result with simulations of the egg model in equilibrium is shown in Fig. 7. Note that the result (A3) is independent of $h, \tau_{\mathrm{B}}$ and $\alpha$. As expected, $S_{2}$ increases monotonically with $\kappa$ since a higher anisotropy constant leads to a better alignment of magnetic moment and easy axis orientation. The limiting behavior is

$$
\begin{array}{ll}
S_{2}=\frac{2 \kappa}{15}+\frac{4 \kappa^{2}}{315}-\frac{8 \kappa^{3}}{4725}-\frac{16 \kappa^{4}}{31185}+\mathcal{O}\left(\kappa^{5}\right) & \text { for } \kappa \ll 1 \\
S_{2}=1-\frac{3}{2 \kappa}-\frac{3}{4 \kappa^{2}}+\frac{15}{16 \kappa^{3}}+\mathcal{O}\left(\kappa^{-4}\right) & \text { for } \kappa \rightarrow \infty
\end{array}
$$

Similarly we can define $S_{4}=\left\langle P_{4}(\mathbf{e} \cdot \mathbf{n})\right\rangle_{\text {eq }}$ with the fourth order Legendre polynomial $P_{4}(x)=\left(35 x^{4}-30 x^{2}+3\right) / 8$. Note that due to the symmetry $\mathbf{n} \rightarrow-\mathbf{n}$, only averages of even Legendre polynomials are non-zero. The integration gives

$$
S_{4}=\frac{5(2 \kappa-21)}{32 \kappa^{3 / 2} \mathrm{Da}(\sqrt{\kappa})}+\frac{3(\kappa+5)}{8 \kappa}+\frac{105}{32 \kappa^{2}}
$$

with the limiting behavior

$$
\begin{array}{ll}
S_{4}=\frac{4 \kappa^{2}}{315}+\frac{16 \kappa^{3}}{10395}+\mathcal{O}\left(\kappa^{4}\right) & \text { for } \kappa \ll 1 \\
S_{4}=1-\frac{5}{\kappa}+\frac{25}{4 \kappa^{2}}+\mathcal{O}\left(\kappa^{-3}\right) & \text { for } \kappa \rightarrow \infty
\end{array}
$$

The increase of $S_{2}$ and $S_{4}$ with $\kappa$ is shown in Fig. 7. Excellent agreement of the numerical solutions with the exact results (A3) and (A6) is found. It is interesting to note that convergence to perfect alignment $S_{2}, S_{4} \rightarrow 1$ for $\kappa \rightarrow \infty$ is rather slow so that even for $\kappa=5$ the order parameter $S_{2} \approx 0.65, S_{4} \approx 0.29$, i.e. significant deviations from perfect alignment that would correspond to the rigid-dipole approximation.

\section{Appendix B: Stochastic formulation of "egg model"}

Our aim here is to find the stochastic differential equations for the random vectors $\mathbf{e}_{t}$ and $\mathbf{n}_{t}$ corresponding to Eq. (3). To this end, we rewrite the Fokker-Planck equation (3) as

$$
\frac{\partial F}{\partial t}=-\mathcal{L}_{e} \cdot\left[\mathbf{A}_{e} F\right]-\mathcal{L}_{n} \cdot\left[\mathbf{A}_{n} F\right]+\frac{1}{2}\left(\begin{array}{c}
\mathcal{L}_{e} \\
\mathcal{L}_{n}
\end{array}\right)\left(\begin{array}{c}
\mathcal{L}_{e} \\
\mathcal{L}_{n}
\end{array}\right): \mathbf{D} F
$$

and identify the drift terms, which in the present case are effective angular velocities,

$$
\begin{aligned}
& \mathbf{A}_{e}=\boldsymbol{\Omega}+\boldsymbol{\omega}_{L}+\frac{1}{2 \tau_{0}} \mathbf{e} \times \mathbf{h}+\frac{1}{\tau_{\mathrm{D}}} \kappa(\mathbf{e} \cdot \mathbf{n}) \mathbf{e} \times \mathbf{n} \\
& \mathbf{A}_{n}=\boldsymbol{\Omega}+\frac{1}{2 \tau_{\mathrm{B}}} \mathbf{e} \times \mathbf{h} .
\end{aligned}
$$

The diffusion matrix $\mathbf{D}$ appearing in Eq. (B1) is given by

$$
\mathbf{D}=\left(\begin{array}{cc}
1 / \tau_{0} \mathbf{I} & 1 / \tau_{\mathrm{B}} \mathbf{I} \\
1 / \tau_{\mathrm{B}} \mathbf{I} & 1 / \tau_{\mathrm{B}} \mathbf{I}
\end{array}\right)
$$




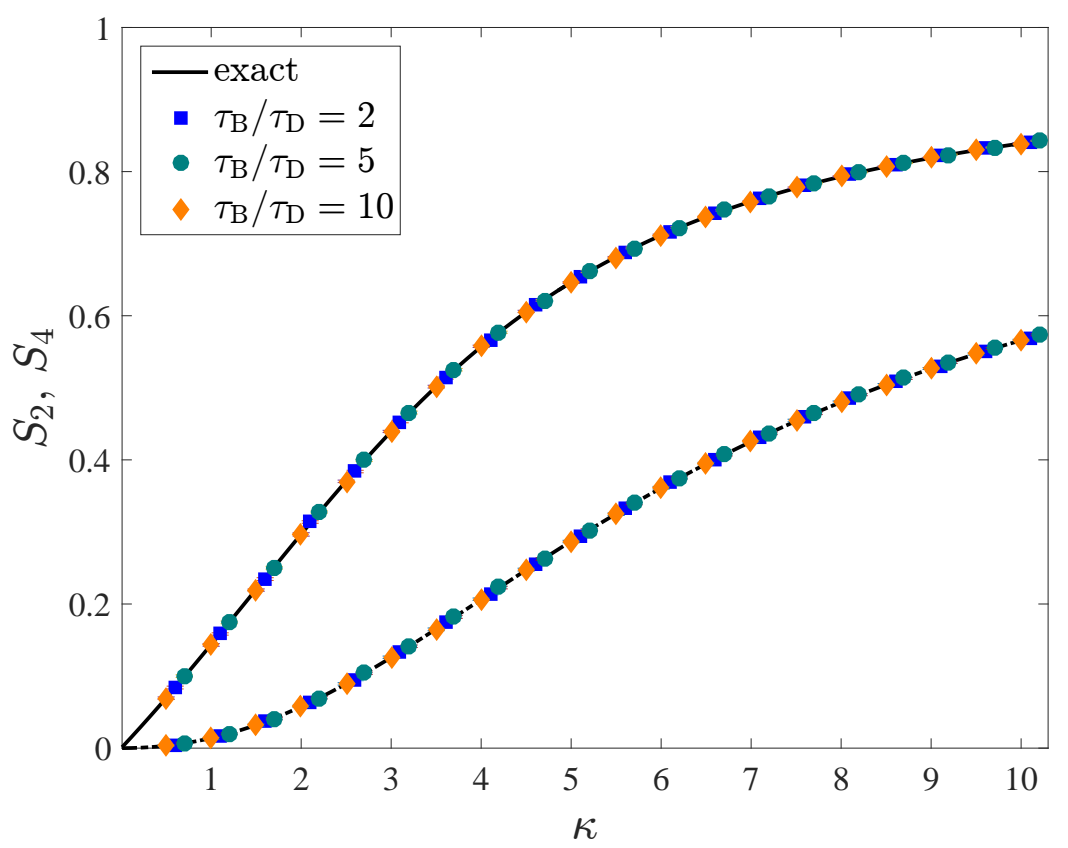

FIG. 7. The alignment order parameters $S_{n}=\left\langle P_{n}(\mathbf{e} \cdot \mathbf{n})\right\rangle_{\text {eq }}$ with $n=2$ (top) and $n=4$ (bottom) as a function of the dimensionless anisotropy energy $\kappa$ for different values of $\tau_{\mathrm{B}} / \tau_{\mathrm{D}}$.

where I denotes the three-dimensional unit matrix.

Equation (B1) is of the general form of the Fokker-Planck equation, where the drift terms can be rewritten due to $-\mathcal{L}_{e} \cdot\left[\mathbf{A}_{e} P\right]=-\frac{\partial}{\partial \mathbf{e}} \cdot\left(\mathbf{A}_{e} \times \mathbf{e} P\right)$ and similarly for $\mathcal{L}_{n}$. In order to exploit the general relation between Fokker-Planck and corresponding stochastic differential equations [24], we decompose the symmetric matrix $\mathbf{D}$ as

$$
\mathbf{D}=\mathbf{B} \cdot \mathbf{B}^{T} .
$$

In order to recover (B4), the matrix $\mathbf{B}$ can be chosen as

$$
\mathbf{B}=\left(\begin{array}{cc}
1 / \sqrt{\tau_{0}} \mathbf{I} & 1 / \sqrt{\tau_{\mathrm{B}}} \mathbf{I} \\
\mathbf{0} & 1 / \sqrt{\tau_{\mathrm{B}}} \mathbf{I}
\end{array}\right)
$$

and $\mathbf{0}$ is the three-dimensional matrix consisting only of zeros. Therefore, the stochastic differential equations for $\mathbf{e}_{t}, \mathbf{n}_{t}$ corresponding to Eq. (B1) read

$$
\left(\begin{array}{c}
\mathrm{d} \mathbf{e}_{t} \\
\mathrm{~d} \mathbf{n}_{t}
\end{array}\right)=-\left(\begin{array}{c}
\mathbf{e}_{t} \times \mathbf{A}_{e} \\
\mathbf{n}_{t} \times \mathbf{A}_{n}
\end{array}\right) \mathrm{d} t-\left(\begin{array}{c}
\mathbf{e}_{t} \times\left[\mathrm{d} \mathbf{W}_{t}^{(e)} / \sqrt{\tau_{0}}+\mathrm{d} \mathbf{W}_{t}^{(n)} / \sqrt{\tau_{\mathrm{B}}}\right] \\
\mathbf{n}_{t} \times \mathrm{d} \mathbf{W}_{t}^{(n)} / \sqrt{\tau_{\mathrm{B}}}
\end{array}\right),
$$

where $\mathbf{W}_{t}^{(e)}, \mathbf{W}_{t}^{(n)}$ are independent, three-dimensional Wiener processes. Inserting the expression for the drift terms (B2), (B3) and the matrix B from Eq. (B6) into (B7) leads to Eqs. (5), (6).

\section{Appendix C: Estimate of Néel relaxation time}

We are interested in the case where the Néel relaxation process can be described as a thermally activated magnetization reversal with spontaneous jumps $\mathbf{e} \rightarrow-\mathbf{e}$ over the anisotropy barrier to populate the energy minima $\mathbf{e}= \pm \mathbf{n}$ parallel to the easy axis of the nanoparticle.

We follow Brown's classical treatment and use transition state theory in order to estimate the effective rate of barrier crossings [10]. To this end, we must first map the Fokker-Planck equation (3) in the variables e, $\mathbf{n}$ onto an effective one-dimensional equation for the reaction coordinate $y$. We choose as reaction coordinate $y=\mathbf{e} \cdot \mathbf{n}$. From the definition $-1 \leq y \leq 1$ and $y<0$ and $y>0$ distinguish the two energy wells corresponding to parallel and anti-parallel alignment. Thus we define the instantaneous probability density of the reaction coordinate

$$
\psi(y ; t)=\iint \delta(\mathbf{e} \cdot \mathbf{n}-y) F(\mathbf{e}, \mathbf{n} ; t) \mathrm{d} \mathbf{n} \mathrm{d} \mathbf{e} .
$$


From Eq. (3) we can derive the time evolution equation for $\psi$ as

$$
\begin{aligned}
\frac{\partial}{\partial t} \psi(y ; t) & =\iint \delta(\mathbf{e} \cdot \mathbf{n}-y) \frac{\partial}{\partial t} F(\mathbf{e}, \mathbf{n} ; t) \mathrm{d} \mathbf{n} \mathrm{d} \mathbf{e}=-\frac{\partial}{\partial y} J_{y}(y ; t) \\
J_{y}(y ; t) & =\iint\left[\mathbf{J}_{e}-\mathbf{J}_{n}\right] \cdot(\mathbf{e} \times \mathbf{n}) \delta(\mathbf{e} \cdot \mathbf{n}-y) \mathrm{d} \mathbf{n} \mathrm{d} \mathbf{e}
\end{aligned}
$$

with

$$
\mathbf{J}_{e}-\mathbf{J}_{n}=\left[\boldsymbol{\omega}_{L}+\frac{1}{2 \tau_{\mathrm{D}}} \mathbf{e} \times \mathbf{h}+\frac{1}{\tau_{\mathrm{D}}} \kappa(\mathbf{e} \cdot \mathbf{n}) \mathbf{e} \times \mathbf{n}\right] F-\frac{1}{2 \tau_{\mathrm{D}}} \mathcal{L}_{e} F
$$

We can distinguish different contributions to the flux $J_{y}$, such as diffusion

$$
\begin{aligned}
J_{y}^{\mathrm{d}}(y ; t) & =\iint\left[-\frac{1}{2 \tau_{\mathrm{D}}} \mathcal{L}_{e} F(\mathbf{e}, \mathbf{n} ; t)\right] \cdot(\mathbf{e} \times \mathbf{n}) \delta(\mathbf{e} \cdot \mathbf{n}-y) \mathrm{d} \mathbf{n} \mathrm{d} \mathbf{e} \\
& =-\frac{1}{\tau_{\mathrm{D}}} y \psi(y ; t)-\frac{1}{2 \tau_{\mathrm{D}}} \partial_{y}\left(1-y^{2}\right) \psi(y ; t),
\end{aligned}
$$

the anisotropy energy,

$$
\begin{aligned}
J_{y}^{\kappa}(y ; t) & =\frac{\kappa}{\tau_{\mathrm{D}}} \iint(\mathbf{e} \cdot \mathbf{n})(\mathbf{e} \times \mathbf{n})^{2} F(\mathbf{e}, \mathbf{n} ; t) \delta(\mathbf{e} \cdot \mathbf{n}-y) \mathrm{d} \mathbf{n} \mathrm{d} \mathbf{e} \\
& =\frac{\kappa}{\tau_{\mathrm{D}}} y\left(1-y^{2}\right) \psi(y ; t),
\end{aligned}
$$

and the contribution of an external magnetic field

$$
\begin{aligned}
J_{y}^{h}(y ; t) & =\iint\left[\boldsymbol{\omega}_{L}+\frac{1}{2 \tau_{\mathrm{D}}} \mathbf{e} \times \mathbf{h}\right] \cdot(\mathbf{e} \times \mathbf{n}) F(\mathbf{e}, \mathbf{n} ; t) \delta(\mathbf{e} \cdot \mathbf{n}-y) \mathrm{d} \mathbf{n} \mathrm{d} \mathbf{e} \\
& =\frac{1}{2 \tau_{\mathrm{D}}} \iint\left[\frac{1}{\alpha} \mathbf{h} \cdot(\mathbf{e} \times \mathbf{n})+\mathbf{h} \cdot \mathbf{n}-(\mathbf{e} \cdot \mathbf{h})(\mathbf{e} \cdot \mathbf{n})\right] F(\mathbf{e}, \mathbf{n} ; t) \delta(\mathbf{e} \cdot \mathbf{n}-y) \mathrm{d} \mathbf{n} \mathrm{d} \mathbf{e} .
\end{aligned}
$$

We can simplify equations (C6) and (C8) as

$$
\tau_{\mathrm{D}}\left(J_{y}^{\mathrm{d}}+J_{y}^{\kappa}\right)=\frac{1}{2}\left(1-y^{2}\right) e^{\kappa y^{2}} \partial_{y}\left[e^{-\kappa y^{2}} \psi\right]
$$

We immediately verify that the flux $J_{y}^{\mathrm{d}}+J_{y}^{\kappa}$ vanishes when evaluated with the equilibrium probability density,

$$
\psi_{0}(y)=C e^{\kappa y^{2}}, \quad C^{-1}=2 \int_{0}^{1} e^{\kappa y^{2}} \mathrm{~d} y=\sqrt{\frac{\pi}{\kappa}} \operatorname{erfi}(\sqrt{\kappa}) .
$$

From Eq (C11) we can follow the usual steps in transition state theory [24] to derive an approximate expression for the probability flux over the barrier for large $\kappa$ as follows:

$$
\begin{aligned}
2 \tau_{\mathrm{D}} e^{-\kappa y^{2}}\left(J_{y}^{\mathrm{d}}+J_{y}^{a}\right) & =\left(1-y^{2}\right) \partial_{y}\left[e^{-\kappa y^{2}} \psi(y ; t)\right] \\
2 \tau_{\mathrm{D}} \int_{-1}^{0} e^{-\kappa y^{2}}\left(J_{y}^{\mathrm{d}}+J_{y}^{a}\right) \mathrm{d} y & =\int_{-1}^{0}\left(1-y^{2}\right) \partial_{y}\left[e^{-\kappa y^{2}} \psi(y ; t)\right] \mathrm{d} y \\
& =\left.\left(1-y^{2}\right) e^{-\kappa y^{2}} \psi\right|_{-1} ^{0}-\int_{-1}^{0}(-2 y) e^{-\kappa y^{2}} \psi(y ; t) \mathrm{d} y \\
& =\psi(0 ; t)-\left.e^{-\kappa y^{2}} \psi\right|_{-1} ^{0} \\
& =e^{-\kappa} \psi(-1 ; t) \\
& \approx e^{-\kappa} \psi_{0}(-1)=C,
\end{aligned}
$$

where, following transition state theory, we assumed that the probability density in the energy minimum, $\psi(-1)$, can be approximated by the equilibrium value $\psi_{0}(-1)$. For the left hand side, original transition state theory uses saddle 
point integration. Here, in a similar spirit we can define

$$
\begin{aligned}
2 \tau_{\mathrm{D}} \int_{-1}^{0} e^{-\kappa y^{2}}\left(J_{y}^{\mathrm{d}}+J_{y}^{a}\right) \mathrm{d} y & =\bar{J}_{y} 2 \tau_{\mathrm{D}} \int_{-1}^{0} e^{-\kappa y^{2}} \mathrm{~d} y \\
& =\bar{J}_{y} \tau_{\mathrm{D}} \sqrt{\frac{\pi}{\kappa}} \operatorname{erf}(\sqrt{\kappa}) .
\end{aligned}
$$

Putting these results together we obtain

$$
\begin{aligned}
& \bar{J}_{y}=\frac{1}{\tau_{\mathrm{D}}} \frac{\kappa / \pi}{\operatorname{erf}(\sqrt{\kappa}) \operatorname{erfi}(\sqrt{\kappa})} \\
& \kappa \gg 1, \frac{1}{\tau_{\mathrm{D}}} \frac{\kappa^{3 / 2}}{\sqrt{\pi} e^{\kappa}}=\frac{1}{2 \tau_{\mathrm{N}}},
\end{aligned}
$$

where the expression for $\tau_{\mathrm{N}}$ agrees with Brown's result for large barriers [10]. Therefore, we find that Brown's expression for the Néel relaxation time is not affected by the coupling to rotational Brownian motion, at least in the absence of external fields.

As a side remark, we note that transition state theory expressions for the flux are usually expressed as $J=\hat{t} k_{\mathrm{TST}}$ with $k_{\mathrm{TST}}=\frac{\omega_{\min }}{2 \pi} e^{-U_{\min } / k_{\mathrm{B}} T}$ and transmission coefficient $\hat{t}=\omega_{\max } / \xi$ with friction coefficient $\xi$ and $\omega_{\max }$ a measure for the second derivative of the potential energy at the maximum. The present results are in agreement with those general expressions for $k_{\mathrm{TST}}=1 / \tau_{\mathrm{N}}$ and $\hat{t}=1 / 2$.

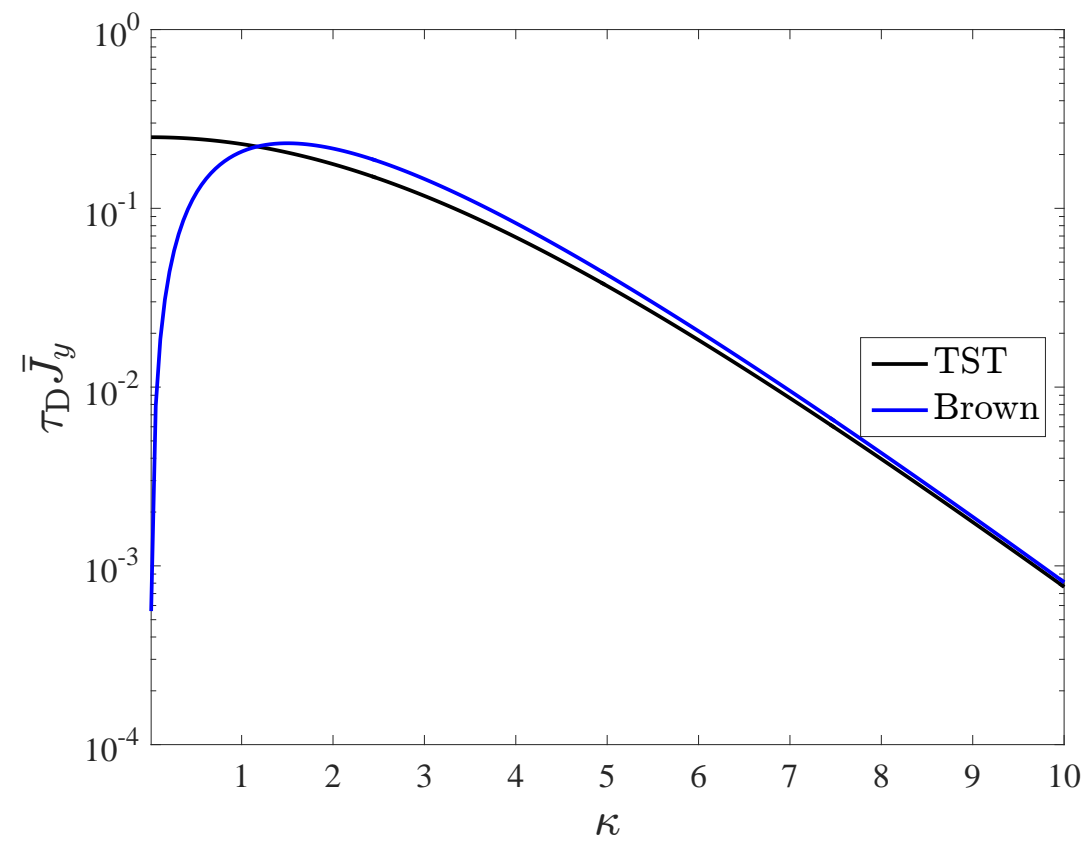

FIG. 8. Probability flux $\bar{J}_{y}$ across the anisotropy barrier, Eq (C21), and asymptotic result for large barriers (C22) due to Brown.

Finally, we want to emphasize that Eq. (C10) shows that an external magnetic field changes the rate $J_{y}$ of barrier crossings in a non-trivial manner and therefore lead to a modification of the effective Néel relaxation time. Unfortunately, the corresponding expression is given only in implicit form. Therefore, we treat the magnetic field-induced modification of the transition rates as an unknown contribution.

\section{Appendix D: Rotational viscosity for kinetic MC model}

In order to calculate the stationary nonequilibrium solution of Eq. (14) for weak flow, $|\boldsymbol{\Omega}| \ll 1$, we use the ansatz

$$
f(\mathbf{e})=f_{\text {eq }}(\mathbf{e})\left[1+\left(\mathbf{e}-\langle\mathbf{e}\rangle_{\text {eq }}\right) \cdot \mathbf{a}\right],
$$


with the equilibrium probability density $f_{\text {eq }}(\mathbf{e})=z_{\text {eq }}^{-1} \exp [\mathbf{e} \cdot \mathbf{h}], z_{\text {eq }}=4 \pi \sinh (h) / h$ and $\langle\bullet\rangle_{\text {eq }}$ here denote averages taken with $f_{\text {eq }}$. We want to determine the unknown vector a to first order in $\boldsymbol{\Omega}$. With Eq. (D1) we can calculate expectation values such as

$$
\begin{aligned}
\langle\mathbf{e}\rangle & =\langle\mathbf{e}\rangle_{\mathrm{eq}}+\left(\langle\mathbf{e e}\rangle_{\mathrm{eq}}-\langle\mathbf{e}\rangle_{\mathrm{eq}}\langle\mathbf{e}\rangle_{\mathrm{eq}}\right) \cdot \mathbf{a} \\
& =L_{1} \hat{\mathbf{h}}+\left(L_{2}-L_{1}^{2}\right)(\mathbf{a} \cdot \hat{\mathbf{h}}) \hat{\mathbf{h}}+\frac{L_{1}}{h} \mathbf{a}
\end{aligned}
$$

where the quantities $L_{n}$ have been defined after Eq. (20) and $\hat{\mathbf{h}}=\mathbf{h} / h$ denotes the unit vector in the direction of the applied field. Similar calculations lead to $\langle\mathbf{e e}\rangle \cdot \hat{\mathbf{h}}=O \hat{\mathbf{h}}+\left(L_{2} / h\right) \mathbf{a}$, where the term $O$ is irrelevant for the rotational viscosity. Details of the calculation can be found e.g. in Ref. [6, 26].

In order to calculate $\left\langle r e^{-\mathbf{e} \cdot \mathbf{h}} \mathbf{e}\right\rangle$ we first note that this quantity vanishes in equilibrium, $\left\langle r e^{-\mathbf{e} \cdot \mathbf{h}} \mathbf{e}\right\rangle_{\mathrm{eq}}=\mathbf{0}$, due to the detailed balance condition (11). Next, due to uniaxial symmetry we have

$$
\left\langle r e^{-\mathbf{e} \cdot \mathbf{h}} \mathbf{e e}\right\rangle_{\mathrm{eq}}=R_{1} \hat{\mathbf{h}} \hat{\mathbf{h}}+R_{2} \mathbf{I}
$$

where the term $R_{1}$ is irrelevant for the rotational viscosity and $R_{2}=\frac{1}{2}\left(I_{0}-I_{2}\right)$ with

$$
\begin{aligned}
I_{n} & =\left\langle(\mathbf{e} \cdot \hat{\mathbf{h}})^{n} r(\mathbf{e} \cdot \mathbf{h}) e^{-\mathbf{e} \cdot \mathbf{h}}\right\rangle_{\mathrm{eq}} \\
& =\frac{1}{2 h^{n} \sinh (h)} \int_{-h}^{h} y^{n} r(y) \mathrm{d} y .
\end{aligned}
$$

Therefore, the ansatz (D1) leads to $\left\langle r e^{-\mathbf{e} \cdot \mathbf{h}} \mathbf{e}\right\rangle=R_{1}(\mathbf{a} \cdot \hat{\mathbf{h}}) \hat{\mathbf{h}}+R_{2} \mathbf{a}$.

Inserting these expressions into Eq. (14) gives an equation for the unknown a and the resulting magnetization from Eq. (D2). For the rotational viscosity, only the component perpendicular to the applied field is of importance and the explicit form of $R_{1}$ is irrelevant. We therefore apply the orthogonal projector $\mathbf{I}-\hat{\mathbf{h}} \hat{\mathbf{h}}$ to Eq. (14) which, in the stationary states, leads to

$$
\mathbf{0}=L_{1} \boldsymbol{\Omega} \times \hat{\mathbf{h}}-\frac{L_{2}+2 L_{1} / h}{2 \tau_{\mathrm{B}}}\left(1+\frac{\tau_{\mathrm{B}}}{\tau_{\mathrm{N}}} \ell(h)\right) \mathbf{a}^{\perp}
$$

where $\mathbf{a}^{\perp}=\mathbf{a}-(\mathbf{a} \cdot \hat{\mathbf{h}}) \hat{\mathbf{h}}$ and $\ell(h)=2 h R_{2} /\left[h-L_{1}\right]$. With $\mathbf{a}^{\perp}$ at hand, the perpendicular magnetization component is determined by $\left\langle\mathbf{e}^{\perp}\right\rangle=\left(L_{1} / h\right) \mathbf{a}^{\perp}$. From Eq. (19) we therefore find the rotational viscosity to be given by

$$
\eta_{\mathrm{rot}}=\eta_{\mathrm{rot}}^{\mathrm{sat}} \frac{h L_{1}^{2}(h)}{h-L_{1}(h)}\left(1+\frac{\tau_{\mathrm{B}}}{\tau_{\mathrm{N}}} \ell(h)\right)^{-1}
$$

where $\eta_{\text {rot }}^{\text {sat }}$ denotes the saturation value of the rotational viscosity. We note that the above derivation leads to the expression $\eta_{\mathrm{rot}}^{\text {sat }}=\frac{3}{2} \eta_{\mathrm{s}} \phi$ which, as discussed in Sect. IV C, is valid only in the rigid-dipole limit. Therefore, we instead use the corrected formula $(22)$ for the saturation value $\eta_{\mathrm{rot}}^{\mathrm{sat}}$.

The function $\ell(h)$ depends on the particular form of the rate $r(x)$. In the simplest case $r(x)=1$ we find $I_{n}=$ $h /[(n+1) \sinh (h)]$ for $n$ even and therefore $R_{2}=h /[3 \sinh (h)]$. More generally, if we $\operatorname{set} r(x)=\cosh (a x)$ with an unknown parameter $a, 0 \leq a \leq 1$, we find $I_{0}=\sinh (a h) /[a \sinh (h)]$. Using a similar calculation for $I_{2}$ and substituting the result we find

$$
\ell_{a}(h)=\frac{2 \sinh (a h) L_{1}(a h)}{a^{2} \sinh (h)\left[h-L_{1}(h)\right]} .
$$

The function $\ell_{a}(h)$ is bounded, $0 \leq \ell \leq 1$ and monotonously decreasing with increasing $h$. The limiting behaviors are $\ell_{a}(h)=1-\left[1-a^{2} / 2\right] h^{2} / 5+\left[41-28 a^{2}+5 a^{4}\right] h^{4} / 1400+\mathcal{O}\left(h^{6}\right)$ for $h \rightarrow 0$ and $\ell_{a}(h) \approx\left(2 / a^{2} h\right) e^{-(1-a) h} \rightarrow 0$ for $h \rightarrow \infty$. Figure 9 shows a plot of the function $\ell_{a}(h)$ for some values of $a$. We include in Fig. 9 also the function $\ell_{\mathrm{G}}(h)$ that corresponds to the choice $r(x)=\operatorname{sech}(x)$ which describes Glauber-like rate functions $\lambda_{\mathrm{G}}=\left(2 \tau_{\mathrm{N}}\right)^{-1}[1+\tanh (-\mathbf{e} \cdot \mathbf{h})]$. Since the corresponding integrals $I_{n}$ from Eq. (D6) lead to cumbersome expressions, we only show here the numerical result for $\ell_{\mathrm{G}}(h)$. We observe that Arrhenius $(a=0)$ and Glauber rates lead to very similar results for $\ell(h)$.

Since the first order expansion of $\ell_{a}$ is independent of $a$, we obtain $\eta_{\text {rot }}=\frac{1}{4} \eta_{\mathrm{s}} \phi q h^{2} \tau_{\mathrm{N}} /\left(\tau_{\mathrm{N}}+\tau_{\mathrm{B}}\right), q=\left(1+3 /\left[2 \kappa S_{2}\right]\right)^{-1}$, for $h \ll 1$ independent of the particular form of the rate factor $r$. The same result for the rotational viscosity in weak fields was obtained in Ref. [13] directly from the egg model for the case $\tau_{\mathrm{B}} \gg \tau_{\mathrm{D}}$ but with $q$ replaced by $q_{0}=\left(1+2 S_{2}\right) / 3$. Since $q, q_{0} \rightarrow 1$ for large $\kappa$, both results asymptotically reduce to the rigid-dipole result. In the 


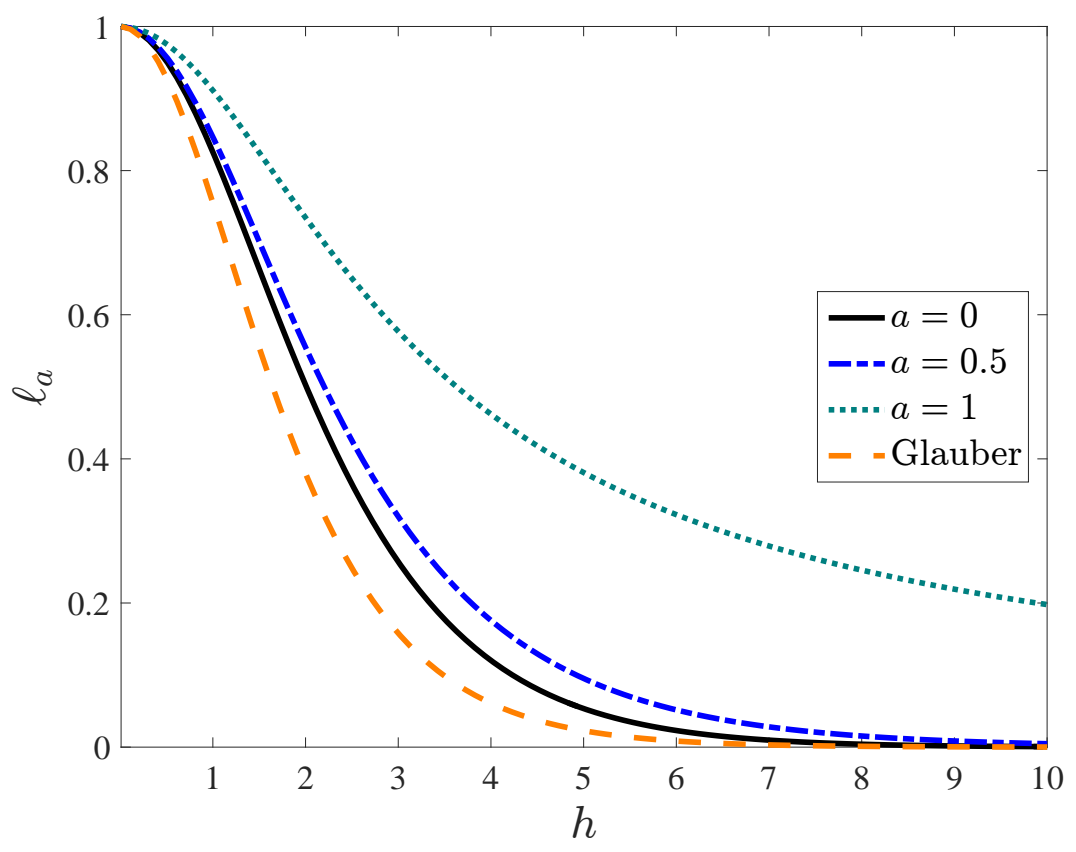

FIG. 9. The dimensionless function $\ell_{a}(h)$ defined in Eq. (D9) for selected values of the parameter $a$ as well $\ell_{\mathrm{G}}$ for Glauber rates.

opposite limit of very strong magnetic fields, Eq. (D8) predicts that the rotational viscosity reaches the asymptotic value $\eta_{\mathrm{rot}}^{\mathrm{sat}}=(3 / 2) \eta_{\mathrm{s}} \phi q$ for $h \rightarrow \infty$. Shliomis and Stepanov [13] found that the rigid-dipole limit value is reduced not by a factor $q$ but $q_{\infty}=35 S_{2}^{2} /\left[16 S_{4}+5 S_{2}+14\right]$. As discussed in [31], both expressions behave very similar for large $\kappa$. It is interesting to note that $q \rightarrow 1$ converges only slowly as the anisotropy constant $\kappa$ is increased (see Fig. 7 ).

[1] E. Blums, A. Cebers, and M. M. Maiorov, Magnetic Fluids (de Gruyter, Berlin, 1997).

[2] P. Ilg and S. Odenbach, in Colloidal Magnetic Fluids: Basics, Development and Applications of Ferrofluids, edited by S. Odenbach (Springer, Berlin, 2008), vol. 763 of Lecture Notes in Phys.

[3] F. Wiekhorst, U. Steinhoff, D. Eberbeck, and L. Trahms, Pharmaceutical research 29, 1189 (2012).

[4] Q. A. Pankhurst, N. T. K. Thanh, S. K. Jones, and J. Dobson, J. Phys. D: Appl. Phys. 42, 224001 (2009).

[5] M. A. Martsenyuk, Y. L. Raikher, and M. I. Shliomis, Sov. Phys. JETP 38, 413 (1974).

[6] P. Ilg and M. Kröger, Phys. Rev. E 66, 021501 (2002), Erratum: Phys. Rev. E, 67:049901, 2003.

[7] M. Kröger, P. Ilg, and S. Hess, J. Phys.: Condens. Matter 15, S1403 (2003).

[8] S. H. L. Klapp, J. Phys.: Condens. Matter 17, R525 (2005).

[9] A. P. Rosa and F. R. Cunha, Physics of Fluids 31, 052006 (2019).

[10] W. T. Coffey, P. J. Cregg, and Y. P. Kalmykov, in Advances in Chemical Physics, edited by I. Prigogine and S. A. Rice (John Wiley \& Sons, 1993), pp. 263-464.

[11] A. Y. Zubarev, JETP 93, 80 (2001).

[12] S. Mahle, P. Ilg, and M. Liu, Phys. Rev. E 77, 016305 (2008).

[13] M. Shliomis and V. Stepanov, J. Magn. Magn. Mater. 122, 196 (1993).

[14] M. Shliomis and V. Stepanov, in Relaxation Phenomena in Condensed Matter, edited by W. Coffey (John Wiley \& Sons, 1994), vol. LXXXVII of Advances in Chemical Physics, pp. 1-30.

[15] M. Suto, Y. Hirota, H. Mamiya, A. Fujita, R. Kasuya, K. Tohji, and B. Jeyadevan, J. Magn. Magn. Mater. 321, 1493 (2009).

[16] S. A. Shah, D. B. Reeves, R. M. Ferguson, J. B. Weaver, and K. M. Krishnan, Phys. Rev. B 92, 094438 (2015).

[17] H. Rogge, M. Erbe, T. M. Buzug, and K. Lüdtke-Buzug, Biomedizinische Technik/Biomedical Engineering 58, 1 (2013).

[18] P. Ilg, Phys. Rev. B 95, 214427 (2017).

[19] D. V. Berkov, N. L. Gorn, and D. Stock, J. Magn. Magn. Mater. 272, E1281 (2004).

[20] D. V. Berkov, N. L. Gorn, and D. Stock, J. Magn. Magn. Mater. 310, e1015 (2007).

[21] S. Ruta, R. Chantrell, and O. Hovorka, Scientific Reports 5, 9090 (2015).

[22] C. Jonasson, V. Schaller, L. Zeng, E. Olsson, C. Frandsen, A. Castro, L. Nilsson, L. K. Bogart, P. Southern, Q. A. Pankhurst, et al., J. Magn. Magn. Mater. 477, 198 (2019). 
[23] A. Y. Zubarev and L. Y. Iskakova, Phys. Rev. E 61, 5415 (2000).

[24] C. Gardiner, Stochastic Methods, Springer Series in Synergetics (Springer-Verlag, Berlin Heidelberg, 2009).

[25] J. L. García-Palacios and F. J. Lázaro, Phys. Rev. B 58, 14937 (1998).

[26] P. Ilg, M. Kröger, and S. Hess, J. Chem. Phys. 116, 9078 (2002).

[27] R. Hergt, S. Dutz, R. Müller, and M. Zeisberger, J. Phys.: Condens. Matter 18, S2919 (2006).

[28] S. Dutz and R. Hergt, Nanotechnology 25, 452001 (2014).

[29] P. C. Fannin, S. W. Charles, and T. Relihan, J. Phys. D: Appl. Phys. 28, 1765 (1995).

[30] V. I. Stepanov, J. Magn. Magn. Mater. 258-259, 442 (2003).

[31] K. Morozov, M. Shliomis, and M. Zahn, Phys. Rev. E 73, 066312 (2006).

[32] S. Odenbach, Int. J. Mod. Phys. B 14, 1615 (2000).

[33] A. Y. Zubarev and L. Y. Iskakova, Magnetohydrodynamics 40, 65 (2004).

[34] A. Montefusco, M. A. Peletier, and H. C. Öttinger, Coarse-graining via the fluctuation-dissipation theorem and largedeviation theory, arXiv:1809.07253v1.

[35] A. O. Ivanov and P. J. Camp, Phys. Rev. E 98, 050602 (2018).

[36] A. Sreekumari and P. Ilg, Phys. Rev. E 88, 042315 (2013).

[37] P. Ilg, M. Kröger, and S. Hess, Phys. Rev. E 71, 031205 (2005). 